Supporting Information

\title{
A Chiral Phosphoramidite Reagent for the Synthesis of INOSITOL PHOSPHATES
}

Estelle Durantie, ${ }^{\dagger}$ Samuel Huwiler, ${ }^{\ddagger}$ Jean-Christophe Leroux, and Bastien Castagner*, ${ }^{\S}$

Institute of Pharmaceutical Sciences

Department of Chemistry and Applied Biosciences ETH Zurich

Vladimir Prelog Weg 3, CH 8093 Zurich (Switzerland)

E-mail: bastien.castagner@mcgill.ca 


\section{Table of Contents}

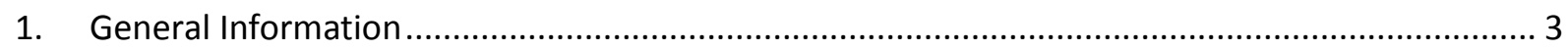

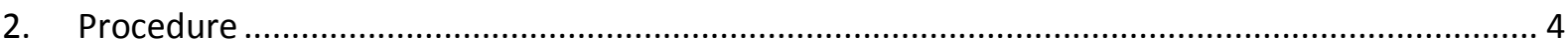

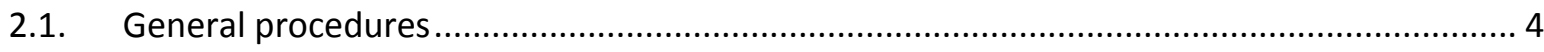

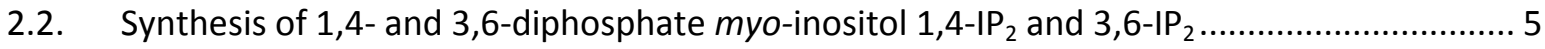

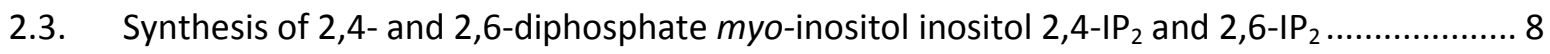

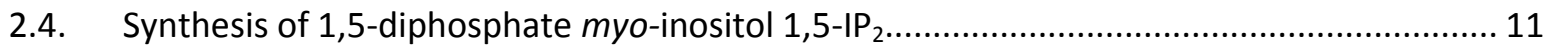

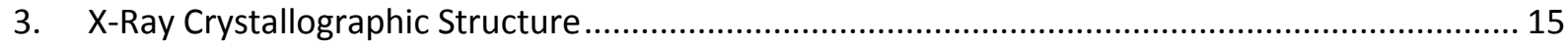

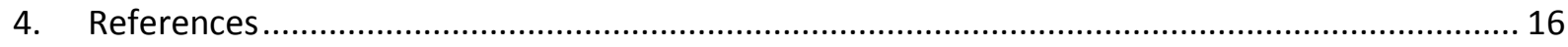

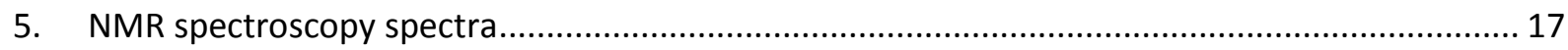




\section{General Information}

Reactions requiring dry conditions were performed under an atmosphere of argon in dried glassware.

All chemicals were reagent grade and used as supplied unless stated otherwise.

Solvents for reactions (diethylether, $\mathrm{N}, \mathrm{N}$-dimethylformamide, methylene chloride, methanol, acetonitrile, acetone) were purchased of analytical grade from commercial suppliers and used without further purification unless stated otherwise. Solvents for extractions and chromatography were technical grade and distilled prior to use.

Ultrapure water was prepared by a Barnstead Nanopure system (Thermo Fischer Scientific, Reinach, Switzerland).

Analytical thin layer chromatography (TLC) was performed on pre-coated Merck silica gel $60 \mathrm{~F}_{254}$ plates and visualized with UV light or cerium molybdate stain.

Flash column chromatography was carried out on Fluka silica gel 60 (230-400 mesh). Ion exchange column chromatography was performed with Dowex ${ }^{\circledR} 50 \mathrm{WX8}-400$ resin (Sigma-Aldrich, St. Louis, USA).

Concentration in vacuo was performed at $\sim 10$ mbar and $40{ }^{\circ} \mathrm{C}$ unless stated otherwise, drying at $\sim 10^{-2} \mathrm{mbar}$ at room temperature $(\mathrm{rt})$.

Lyophilizations were performed on a Christ Freeze Dryer Alpha 2-4 LSC (Birsfelden, Switzerland).

${ }^{1} \mathrm{H},{ }^{13} \mathrm{C},{ }^{19} \mathrm{~F}$ and ${ }^{31} \mathrm{P}$ NMR spectra were recorded on a Bruker $A V 400 \mathrm{MHz}$ spectrometer or a Bruker $A V 500 \mathrm{MHz}$ spectrometer. NMR data are reported as follows: chemical shifts $(\delta, \mathrm{ppm}$, relative to residual solvent peaks), integration, multiplicity $(s=$ singlet, $b r=b r o a d, d=$ doublet, $t=$ triplet, $q=$ quartet, $m=$ multiplet), coupling constant $(J, \mathrm{~Hz})$.

High-resolution mass spectra (HR ESI) were performed by the MS service at the Laboratory for Organic Chemistry, ETH Zurich.

Optical rotations were obtained using a JASCO P-2000 polarimeter. Optical rotations are reported as follows: $[\alpha]^{\top}$ ( $c=1.00$ corresponds to $10.0 \mathrm{mg} \cdot \mathrm{mL}^{-1}$, solvent) with $T=$ temperature of the measurement in ${ }^{\circ} \mathrm{C}$. 


\section{Procedure}

\subsection{General procedures}

\section{Phosphorylation reaction}

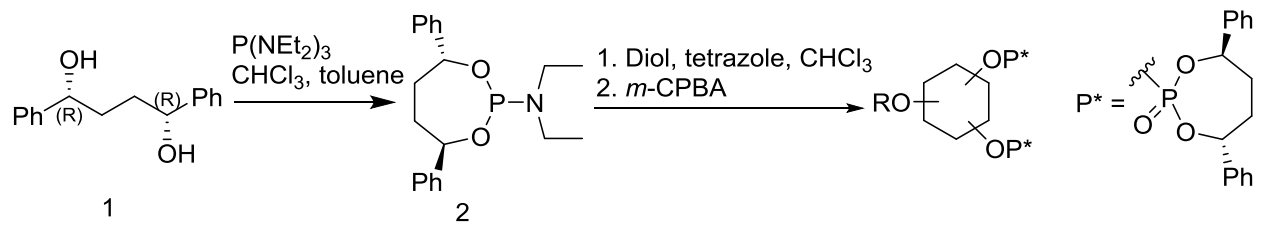

Procedure A: All the solvents used were dried and degassed and the glassware was flame-dried under vacuum followed by argon flush - purge.

In situ preparation of $(1 R, 4 R)-1,4$-diphenylbutane- $N, N$-diethylphosphoramidite 2: A solution of $(1 R, 4 R)$-1,4-diphenylbutandiol $\mathbf{1}^{1,2}$ in $\mathrm{CHCl}_{3}(5.5$ equiv, $1 \mathrm{M})$ was added to boiling toluene. The hexaethyl phosphorous triamide solution in toluene (5.5 equiv, $1 \mathrm{M}$ ) was then added dropwise over a period of $30 \mathrm{~min}$ and then stirred for a further $10 \mathrm{~min}$. An aliquot was taken to verify the purity by ${ }^{1} \mathrm{H}$ and ${ }^{31} \mathrm{P} N M R$.

The solution of tetrazole in acetonitrile $(\mathrm{ACN})(0.45 \mathrm{M}, 23$ equiv) was added to the in situ prepared phosphoramidite 2, followed by addition of the solution of inositol in $\mathrm{CHCl}_{3}$ (1 equiv, $0.02 \mathrm{M}$ ). The reaction mixture was stirred at $\mathrm{rt}$ for $20 \mathrm{~h}$. A solution of $m$-CPBA (75\% w/w, 10 equiv) in $\mathrm{CH}_{2} \mathrm{Cl}_{2}$ was dried over $\mathrm{Na}_{2} \mathrm{SO}_{4}$, added at $-10{ }^{\circ} \mathrm{C}$ to the reaction mixture and stirred at $\mathrm{rt}$ for an additional $45 \mathrm{~min}$. The mixture was then diluted in EtOAc, treated with solution of aqueous $\mathrm{Na}_{2} \mathrm{SO}_{3}$ and washed with a saturated solution of aqueous $\mathrm{NaHCO}_{3}$ and with brine. The organic phase was dried over $\mathrm{Na}_{2} \mathrm{SO}_{4}$, filtered and evaporated to dryness. The obtained residue was purified by flash chromatography as indicated.

\section{Full deprotection using $\mathrm{TMSBr}$}

Procedure B: Protected inositol phosphate (1 equiv) in MeOH/DCM 30\% (0.05 M) was treated with TMSBr (56 equiv) and stirred for $2 \mathrm{~h}$. The reaction mixture was then degassed with $\mathrm{N}_{2}$ and the evacuated $\mathrm{HBr}$ was neutralized in a gas trap with $1 \mathrm{M} \mathrm{NaOH}$ solution. After 1 to $2 \mathrm{~h}$, the reaction mixture was concentrated to dryness. The crude was triturated with acetone and then with ACN to afford clean bisphosphate. 
Ion exchange to afford the $\mathrm{Li}^{+}$salt

Procedure C: Dowex $\mathrm{H}^{+}$was equilibrated to $\mathrm{Li}^{+}$by passing an aqueous solution of $\mathrm{LiOH} 2 \mathrm{M}$ and washed with nanopure $\mathrm{H}_{2} \mathrm{O}$ until obtaining a neutral $\mathrm{pH}$. The solution of inositol phosphate was then loaded on the column to afford the $\mathrm{Li}^{+}$salt which was further dried by lyophilization.

\subsection{Synthesis of 1,4- and 3,6-diphosphate myo-inositol 1,4-IP $\mathrm{P}_{2}$ and $3,6-I \mathrm{P}_{2}$}

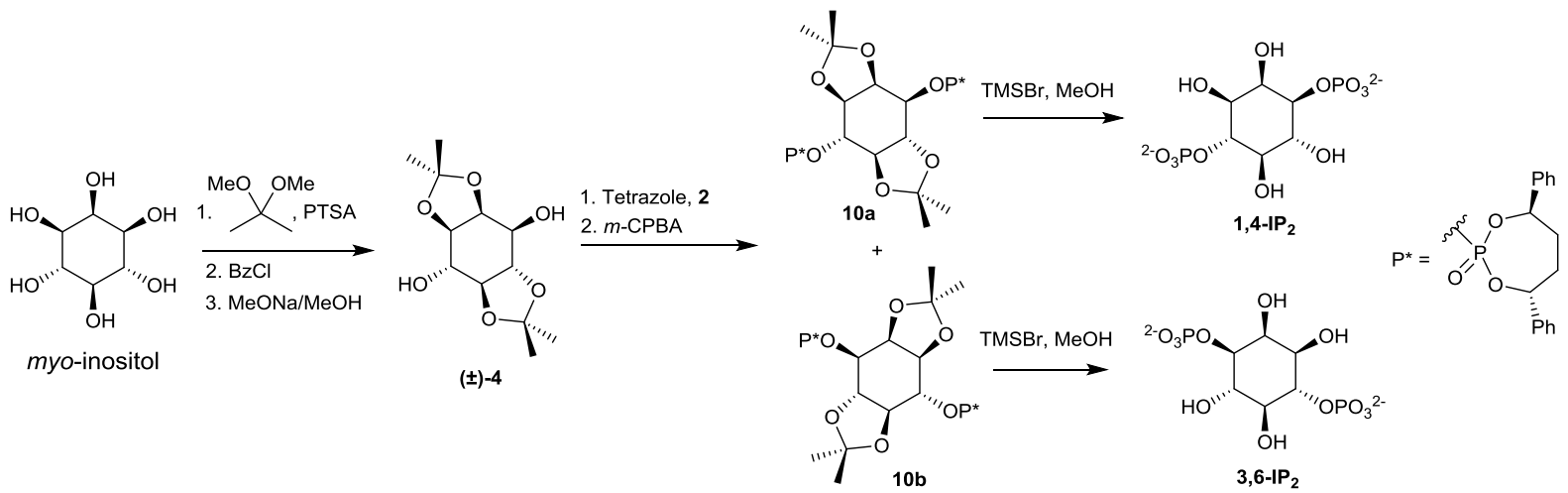

Synthesis of ( \pm )-1,2;5,6-di-O-isopropylidene-myo-inositol ( \pm )-4

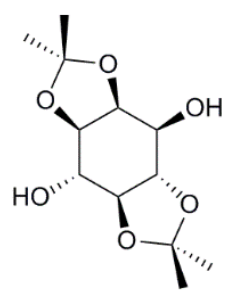

(士) -4

( \pm )-4 was prepared following the procedure described by Kheronsky et. al. ${ }^{3} ;{ }^{1} \mathrm{H}$ NMR spectrum was in accordance with literature.

Synthesis of the 2,3:5,6-di-isoproylidene-1,4-(di-O-(1R,4R)-1,4-diphenylbutanephospho) myoinositol 10a and the 1,2:4,5-di-isoproylidene-3,6-(di-O-(1R,4R)-1,4-diphenylbutanephospho) myoinositol 10b

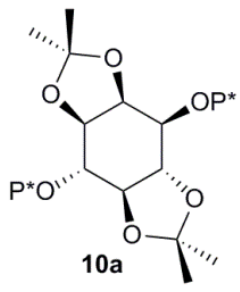

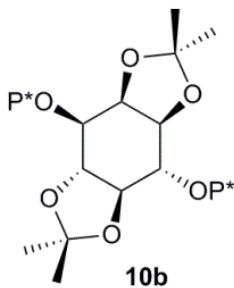

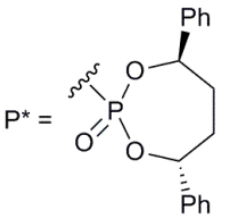

Synthesized from ( \pm )-2,3:5,6-di-isoproylidene-myo-inositol ( \pm )-4 following procedure $A$. The obtained residue was purified by flash chromatography $\left(\mathrm{SiO}_{2}\right.$, EtOAc/hexane $\left.30 \rightarrow 40 \%\right)$ to afford the 
diastereomeric mixture $10 \mathrm{a}$ and $10 \mathrm{~b}$ as white solid (64 mg, 87\%). Crystallization of the mixture by slow evaporation method in $\mathrm{iPrOH}$ afforded diastereomer $10 \mathrm{a}\left(\mathrm{dr}>99: 1,16 \%^{[\mathrm{ad}]}\right)$. Diastereomer $10 \mathrm{~b}$ was obtained by recrystallization of the resulting mother liquor in $i \mathrm{PrOH}\left(\mathrm{dr}>99: 1,13 \%{ }^{[\mathrm{a}]}\right)$.

[a] Yield based on the diastereomer itself, which represent $50 \%$ of the racemic starting material.

10a: $[\alpha]_{D}^{25}+29.2\left(c\right.$ 0.26, $\left.\mathrm{CHCl}_{3}\right) ;{ }^{1} \mathrm{H}$ NMR $\left(400 \mathrm{MHz}, \mathrm{CDCl}_{3}\right) \delta$ (ppm) 7.28-7.42 (20H, m, Ph), 5.53-5.61 $(2 \mathrm{H}, \mathrm{m}, \mathrm{CH}-\mathrm{Ph}), 5.42$ (1H, dd, J 10.0, 7.2 Hz, CH-Ph), 5.34 (1H, dd, J 9.1, 7.6 Hz, CH-Ph), 4.66 (1H, ddd, J 9.9, $\left.{ }^{3} J_{\mathrm{HP}} 8.8, J 4.1 \mathrm{~Hz}, \mathrm{H}-\mathrm{C} 3\right), 4.53-4.61(2 \mathrm{H}, \mathrm{m}, \mathrm{H}-\mathrm{C} 6, \mathrm{H}-\mathrm{C} 2), 4.17(1 \mathrm{H}, \mathrm{dd}, J 6.2,4.6 \mathrm{~Hz}, \mathrm{H}-\mathrm{C} 1), 3.92$ $(1 \mathrm{H}, \mathrm{t}, J 9.9 \mathrm{~Hz}, \mathrm{H}-\mathrm{C} 4), 3.25(1 \mathrm{H}, \mathrm{t}, J 10.1 \mathrm{~Hz}, \mathrm{H}-\mathrm{C} 5), 2.20-2.39\left(4 \mathrm{H}, \mathrm{m}, \mathrm{CH}_{2}\right), 2.04-2.17\left(4 \mathrm{H}, \mathrm{m}, \mathrm{CH}_{2}\right)$, $1.51(3 \mathrm{H}, \mathrm{s}, i \mathrm{Pr}), 1.30(3 \mathrm{H}, \mathrm{s}, i \mathrm{Pr}), 0.88(3 \mathrm{H}, \mathrm{s}, i \mathrm{Pr}), 0.70(3 \mathrm{H}, \mathrm{s}, i \mathrm{Pr}) ;{ }^{13} \mathrm{C} \mathrm{NMR}\left(126 \mathrm{MHz}, \mathrm{CDCl}_{3}\right) \delta(\mathrm{ppm})$ 141.5 (d, $\left.{ }^{3} J_{\mathrm{CP}} 10.9 \mathrm{~Hz}, i \mathrm{Ph}\right), 141.1$ (d, $\left.{ }^{3}{ }_{\mathrm{CP}} 10.6 \mathrm{~Hz}, i \mathrm{Ph}\right), 140.4$ (d, $\left.{ }^{3} \mathrm{JP}_{\mathrm{CP}} 9.9 \mathrm{~Hz}, i \mathrm{Ph}\right), 140.2$ (d, ${ }^{3} \mathrm{JP}_{\mathrm{CP}} 10.0 \mathrm{~Hz}$, $i \mathrm{Ph}), 128.6$ (2xPh), $128.54(\mathrm{Ph}), 128.47$ (2xPh), 128.40 (2xPh), 128.37 (2xPh), $128.3(\mathrm{Ph}), 128.0(\mathrm{Ph})$, $127.9(\mathrm{Ph}), 126.0(2 \times \mathrm{Ph}), 125.9(2 \times \mathrm{Ph}), 125.8(2 \times \mathrm{Ph}), 125.6(2 \times \mathrm{Ph}), 113.0\left(\mathrm{C}\left(\mathrm{CH}_{3}\right)_{2}\right), 111.1\left(\mathrm{C}\left(\mathrm{CH}_{3}\right)_{2}\right)$, $80.5\left(d,{ }^{2} J_{\mathrm{CP}} 6.0 \mathrm{~Hz}, \mathrm{CH}-\mathrm{Ph}\right), 80.2\left(\mathrm{~d},{ }^{2} \mathrm{JPP}_{\mathrm{CP}} 6.0 \mathrm{~Hz}, \mathrm{CH}-\mathrm{Ph}\right), 80.1\left(\mathrm{~d},{ }^{3} \mathrm{~J}_{\mathrm{CP}} 4.2 \mathrm{~Hz}, \mathrm{C} 1\right), 79.8\left(\mathrm{~d},{ }^{2} \mathrm{~J}_{\mathrm{CP}} 2.6 \mathrm{~Hz}, \mathrm{CH}-\right.$ Ph), $79.6\left(d,{ }^{2} J_{C P} 4.4 \mathrm{~Hz}, \mathrm{C} 6\right), 79.2\left(d,{ }^{2} J_{C P} 2.5 \mathrm{~Hz}, \mathrm{CH}-\mathrm{Ph}\right), 76.8(\mathrm{C} 2), 76.5$ (d, $\left.{ }^{3} J_{\mathrm{CP}} 3.3 \mathrm{~Hz}, \mathrm{C} 5\right), 74.9$ (d, ${ }^{3} J_{\mathrm{CP}}$ $6.3 \mathrm{~Hz}, \mathrm{C} 4), 73.9\left(\mathrm{~d},{ }^{2} \mathrm{JP}_{\mathrm{CP}} 3.6 \mathrm{~Hz}, \mathrm{C} 3\right), 38.5\left(\mathrm{CH}_{2}\right), 38.1\left(\mathrm{CH}_{2}\right), 37.5\left(\mathrm{CH}_{2}\right), 37.1\left(\mathrm{CH}_{2}\right), 28.0\left(\mathrm{CH}_{3}\right), 26.2$ $\left(\mathrm{CH}_{3}\right), 26.1\left(\mathrm{CH}_{3}\right), 25.9\left(\mathrm{CH}_{3}\right) ;{ }^{31} \mathrm{P}$ NMR $\left(162 \mathrm{MHz},{ }^{1} \mathrm{H}\right.$-decoupled, $\left.\mathrm{CDCl}_{3}\right) \delta(\mathrm{ppm})-0.72(1 \mathrm{P}),-1.51(1 \mathrm{P})$; $\left[m / z(E S I)(M+H)^{+} C_{44} H_{51} \mathrm{O}_{12} \mathrm{P}_{2}\right.$ requires 833.2850, found 833.2842].

10b: $[\alpha]_{D}^{25}+9.8\left(c 0.17, \mathrm{CHCl}_{3}\right) ;{ }^{1} \mathrm{H}$ NMR (400 MHz, CDCl$) \delta(\mathrm{ppm})$ 7.27-7.45 (20H, m, Ph), 5.53-5.61 $(2 \mathrm{H}, \mathrm{m}, \mathrm{CH}-\mathrm{Ph}), 5.36(1 \mathrm{H}, \mathrm{t}, J 8.6 \mathrm{~Hz}, \mathrm{CH}-\mathrm{Ph}), 5.29(1 \mathrm{H}, \mathrm{t}, J 8.4 \mathrm{~Hz}, \mathrm{CH}-\mathrm{Ph}), 4.80\left(1 \mathrm{H}, \mathrm{ddd}, J 11.0,{ }^{3} J_{\mathrm{HP}}\right.$ 6.8, J $4.3 \mathrm{~Hz}, \mathrm{H}-\mathrm{C} 3), 4.54\left(1 \mathrm{H}, \mathrm{ddd}, J\right.$ 10.5, 9.0, $\left.{ }^{3} \mathrm{JP}_{\mathrm{HP}} 6.8 \mathrm{~Hz}, \mathrm{H}-\mathrm{C} 6\right), 4.35(1 \mathrm{H}, \mathrm{t}, J 4.5 \mathrm{~Hz}, \mathrm{H}-\mathrm{C} 2), 4.03(1 \mathrm{H}$, t, J $9.9 \mathrm{~Hz}, \mathrm{H}-\mathrm{C} 4), 3.99-4.03(1 \mathrm{H}, \mathrm{m}, \mathrm{H}-\mathrm{C} 1), 3.46(1 \mathrm{H}, \mathrm{dd}, J$ J 10.3, $9.9 \mathrm{~Hz}, \mathrm{H}-\mathrm{C} 5), 2.20-2.36\left(4 \mathrm{H}, \mathrm{m}, \mathrm{CH}_{2}\right)$, 2.02-2.19 (4H, m, CH $), 1.43(3 \mathrm{H}, \mathrm{s}, i \operatorname{Pr}), 1.39(3 \mathrm{H}, \mathrm{s}, i \operatorname{Pr}), 1.14(3 \mathrm{H}, \mathrm{s}, i \operatorname{Pr}), 0.52(3 \mathrm{H}, \mathrm{s}, i \operatorname{Pr}) ;{ }^{13} \mathrm{C}$ NMR $\left(126 \mathrm{MHz}, \mathrm{CDCl}_{3}\right) \delta(\mathrm{ppm}) 141.2\left(\mathrm{~d},{ }^{3} \mathrm{JP}_{\mathrm{CP}} 7.4 \mathrm{~Hz}, i \mathrm{Ph}\right), 141.1$ (d, $\left.{ }^{3} J_{\mathrm{CP}} 7.6 \mathrm{~Hz}, i \mathrm{Ph}\right), 140.4$ (d, ${ }^{3} \mathrm{JP}_{\mathrm{CP}} 9.9 \mathrm{~Hz}$, $i \mathrm{Ph}), 140.2\left(\mathrm{~d},{ }^{3} J_{\mathrm{CP}} 10.0 \mathrm{~Hz}, i \mathrm{Ph}\right), 128.6(4 \times \mathrm{Ph}), 128.5(2 \times \mathrm{Ph}), 128.42(\mathrm{Ph}), 128.40(2 \times \mathrm{Ph}), 128.3(\mathrm{Ph})$,

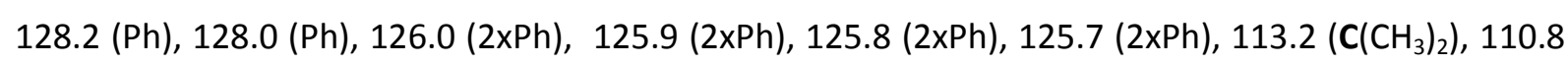
$\left(\mathrm{C}\left(\mathrm{CH}_{3}\right)_{2}\right), 80.53\left(\mathrm{~d},{ }^{2} J_{\mathrm{CP}} 3.8 \mathrm{~Hz}, \mathrm{CH}-\mathrm{Ph}\right), 80.48\left(\mathrm{~d},{ }^{2} \mathrm{~J}_{\mathrm{CP}} 3.5 \mathrm{~Hz}, \mathrm{CH}-\mathrm{Ph}\right), 80.2\left(\mathrm{~d},{ }^{2} \mathrm{JP}_{\mathrm{CP}} 4.4 \mathrm{~Hz}, \mathrm{C} 6\right), 79.8(\mathrm{~d}$, $\left.{ }^{3} J_{\mathrm{CP}} 3.9 \mathrm{~Hz}, \mathrm{C} 1\right), 79.6\left(\mathrm{~d},{ }^{2} \mathrm{JPP}_{\mathrm{CP}} 2.8 \mathrm{~Hz}, \mathrm{CH}-\mathrm{Ph}\right),, 79.2\left(\mathrm{~d},{ }^{2} J_{\mathrm{CP}} 2.6 \mathrm{~Hz}, \mathrm{CH}-\mathrm{Ph}\right), 76.7\left(\mathrm{~d},{ }^{3} \mathrm{JPP}_{\mathrm{CP}} 3.6 \mathrm{~Hz}, \mathrm{C} 5\right), 76.4$ (C2), $75.3\left(\mathrm{~d},{ }^{3} J_{\mathrm{CP}} 7.9 \mathrm{~Hz}, \mathrm{C} 4\right), 74.6\left(\mathrm{~d},{ }^{2}{ }_{\mathrm{CP}} 4.0 \mathrm{~Hz}, \mathrm{C} 3\right), 38.3\left(\mathrm{CH}_{2}\right), 38.2\left(\mathrm{CH}_{2}\right), 37.3\left(\mathrm{CH}_{2}\right), 37.1\left(\mathrm{CH}_{2}\right)$, $28.4\left(\mathrm{CH}_{3}\right), 27.1\left(\mathrm{CH}_{3}\right), 27.0\left(\mathrm{CH}_{3}\right), 24.7\left(\mathrm{CH}_{3}\right) ;{ }^{31} \mathrm{P} \mathrm{NMR}\left(162 \mathrm{MHz},{ }^{1} \mathrm{H}\right.$-decoupled, $\left.\mathrm{CDCl}_{3}\right) \delta(\mathrm{ppm})-1.53$ (1P), $-1.76(1 \mathrm{P}) ;\left[\mathrm{m} / \mathbf{z}(\mathrm{ESI})(\mathrm{M}+\mathrm{H})^{+} \mathrm{C}_{44} \mathrm{H}_{51} \mathrm{O}_{12} \mathrm{P}_{2}\right.$ requires 833.2850, found 833.2849]. 

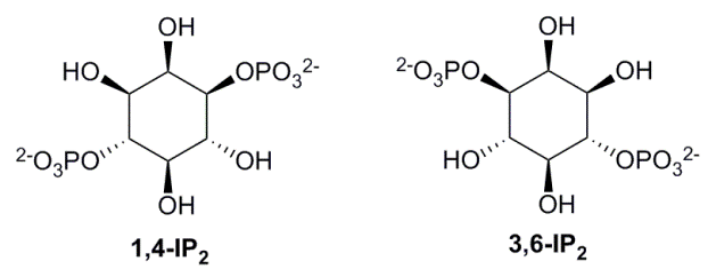

Synthesized from 10a and 10b according to procedure B, except amount of $\operatorname{TMSBr}$ (21 equiv), reaction time $2 \mathrm{~h}$.

1,4-IP $\mathbf{P}_{\mathbf{2}}$ was obtained as a white solid (1.9 $\left.\mathrm{mg}, 5.7 \mu \mathrm{mol}, 89 \%\right) ; \mathbf{3 , 6}-\mathrm{IP}_{\mathbf{2}}$ was obtained as a white solid (0.5 mg, $1.49 \mu \mathrm{mol}, 41 \%$ ).

${ }^{1} \mathrm{H}$ NMR (400 MHz, MeOD) $\delta(\mathrm{ppm}) 4.35(1 \mathrm{H}, \mathrm{q}, J 9.2 \mathrm{~Hz}, \mathrm{H}-\mathrm{C} 6), 4.19(1 \mathrm{H}, \mathrm{t}, J 2.6 \mathrm{~Hz}, \mathrm{H}-\mathrm{C} 2), 4.04(1 \mathrm{H}$, ddd, J 9.8, $\left.{ }^{3} J_{\mathrm{HP}} 8.5, J 2.6 \mathrm{~Hz}, \mathrm{H}-\mathrm{C} 3 / 1\right), 3.86(1 \mathrm{H}, \mathrm{t}, J 9.5 \mathrm{~Hz}, \mathrm{H}-\mathrm{C} 6 / 4), 3.60$ (1H, dd, J 9.6, $\left.2.6 \mathrm{~Hz}, \mathrm{H}-\mathrm{C} 1 / 3\right)$, $3.42(1 \mathrm{H}, \mathrm{t}, J 9.2 \mathrm{~Hz}, \mathrm{H}-\mathrm{C} 5) ;{ }^{13} \mathrm{C}$ NMR $(101 \mathrm{MHz}, \mathrm{MeOD}) \delta(\mathrm{ppm}) 81.3\left(\mathrm{~d},{ }^{2} J_{\mathrm{CP}} 6.1 \mathrm{~Hz}, \mathrm{C} 6 / 4\right), 78.8\left(\mathrm{~d},{ }^{2} \mathrm{CP}_{\mathrm{CP}}\right.$ $5.7 \mathrm{~Hz}, \mathrm{C} 3 / 1$ ), 75.0 (d, ${ }^{3} \mathrm{~J}_{\mathrm{CP}} 2.2 \mathrm{~Hz}, \mathrm{C} 5$ ), 72.6-72.7 (2C, m, C2, C4/6), 72.0 (d, ${ }^{3} \mathrm{JP}_{\mathrm{CP}} 3.2 \mathrm{~Hz}, \mathrm{H}-\mathrm{C} 1 / 3$ ); ${ }^{31}$ P NMR (162 MHz, ${ }^{1} \mathrm{H}$-decoupled, MeOD) $\delta$ (ppm) 1.25 (1P), -0.07 (1P); [m/z (ESI) (M+H) ${ }^{+} \mathrm{C}_{6} \mathrm{H}_{15} \mathrm{O}_{12} \mathrm{P}_{2}$ requires 341.0033 , found 341.0037$]$.

1,4-IP $:[\alpha]_{D}^{23}-4.55$ (c 0.124, $\mathrm{H}_{2} \mathrm{O}, \mathrm{pH}$ 7); $\left\{\text { lit. }[\alpha]_{D}^{22}+1.6 \text { (c 1.3, } \mathrm{H}_{2} \mathrm{O} \text {, free acid) }\right\}^{4[\mathrm{~b}]}$

3,6-IP $2:[\alpha]_{D}^{23}+7.95\left(c 0.029, \mathrm{H}_{2} \mathrm{O}, \mathrm{pH} 7\right) ;\left\{\text { lit. }[\boldsymbol{\alpha}]_{D}^{22}-1.4 \text { (c 1.8, } \mathrm{H}_{2} \mathrm{O} \text {, free acid) }\right\}^{4[b]}$

[b] The optical rotations measured are opposite to the known optical rotation reported in the literature, but measurements have been repeated several times giving similar values. As mentioned by Miller et al., ${ }^{5}$ that have encountered the same phenomenon, optical rotations of inositol phosphates have low magnitude and are dependent on sample preparation (i.e. counter ion, $\mathrm{pH}$ ). In the present case, optical rotation of each compound was measured on the sample without any further preparation, thus it is assumed to be the free acid form, however due to the low concentration, the $\mathrm{pH}$ was neutral. Nonetheless, the absolute configuration is certain as it has been determined by X-Ray analysis in the previous step (compound 10a). 


\subsection{Synthesis of 2,4- and 2,6-diphosphate myo-inositol inositol $2,4-I P_{2}$ and 2,6-IP}

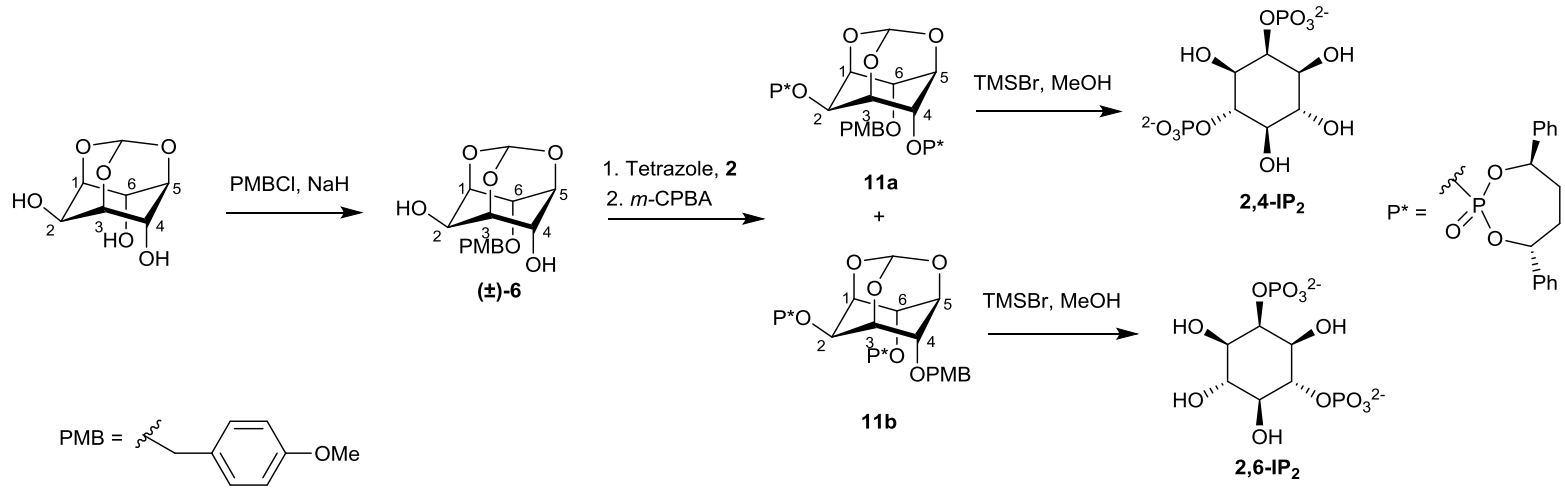

Synthesis of ( \pm )-6-O-(p-methoxybenzyl)-myo-inositol orthoformate ( \pm )-6

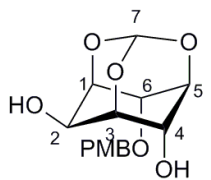

(士) -6

To a solution of myo-inositol orthoformate ( $200 \mathrm{mg}, 1.05 \mathrm{mmol}, 1$ equiv) in DMF dry (7.5 mL, $0.14 \mathrm{M}$ ) at $0{ }^{\circ} \mathrm{C}$ was added portionwise $\mathrm{NaH}(60 \%$ in oil $\mathrm{w} / \mathrm{w} ; 42 \mathrm{mg}, 1.06 \mathrm{mmol}, 1.01$ equiv). The reaction mixture was then treated with $\mathrm{PMBCl}(153 \mu \mathrm{L}, 1.16 \mathrm{mmol}, 1.1$ equiv) and stirred at $\mathrm{rt}$ for $4 \mathrm{~h}$. The mixture was neutralized with $\mathrm{MeOH}$ and concentrated in vacuo at $60{ }^{\circ} \mathrm{C}$. The crude was diluted with EtOAc and washed with $\mathrm{H}_{2} \mathrm{O}$ and then with brine. The organic phase was dried over $\mathrm{Na}_{2} \mathrm{SO}_{4}$ filtered and concentrated in vacuo. Purification by flash column chromatography $\left(\mathrm{SiO}_{2}, \mathrm{EtOAc} /\right.$ hexane $\left.50 \%\right)$ afforded ( \pm )- 6 as a colorless oil ( $244 \mathrm{mg}, 0.78 \mathrm{mmol}, 75 \%)$.

${ }^{1} \mathrm{H}$ NMR $\left(400 \mathrm{MHz}, \mathrm{CDCl}_{3}\right) \delta(\mathrm{ppm}) 7.23(2 \mathrm{H}, \mathrm{d}, J 8.7 \mathrm{~Hz}, \mathrm{Ph}), 6.90(2 \mathrm{H}, \mathrm{d}, J 8.7 \mathrm{~Hz}, \mathrm{Ph}), 5.43(1 \mathrm{H}, \mathrm{d}$, J $1.3 \mathrm{~Hz}, \mathrm{H}-\mathrm{C} 7), 4.61\left(1 \mathrm{H}, \mathrm{d}, J 11.3 \mathrm{~Hz}, \mathrm{CH}_{2}-\mathrm{Ph}\right), 4.57\left(1 \mathrm{H}, \mathrm{d}, J 11.3 \mathrm{~Hz}, \mathrm{CH}_{2}-\mathrm{Ph}\right), 4.44(1 \mathrm{H}, \mathrm{dtd}, J$ 10.3, 3.9, $2.1 \mathrm{~Hz}, \mathrm{H}-\mathrm{C} 6), 4.40(1 \mathrm{H}, \mathrm{td}, J$ 3.9, $2.0 \mathrm{~Hz}, \mathrm{H}-\mathrm{C} 4), 4.23-4.25$ (1H, m, H-C5), 4.18-4.22 (2H, m, H-C3, $\mathrm{H}-\mathrm{C} 1), 4.06(1 \mathrm{H}, \mathrm{d}, J 11.8 \mathrm{~Hz}, \mathrm{H}-\mathrm{C} 2), 3.81\left(3 \mathrm{H}, \mathrm{s}, \mathrm{O}-\mathrm{CH}_{3}\right), 3.77(1 \mathrm{H}, \mathrm{d}, J 10.3 \mathrm{~Hz}, \mathrm{OH}-\mathrm{C} 6), 3.23(1 \mathrm{H}, \mathrm{d}$, J $11.8 \mathrm{~Hz}, \mathrm{OH}-\mathrm{C} 2) ;{ }^{13} \mathrm{C}$ NMR (126 MHz, CDCl ${ }_{3}$ ) (ppm) 160.1 (iPh), 129.9 (2xPh), 127.9 (iPh), 114.3 (2xPh), 102.7 (C7), 74.8 (C1), 73.8 (C4), 72.8 ( $\mathrm{CH}_{2}-\mathrm{Ph}$ ), 72.3 (C3), 67.9 (C6), 67.3 (C5), 60.7 (C2), 55.3 $\left(\mathrm{O}-\mathrm{CH}_{3}\right) ;\left[\boldsymbol{m} / \mathbf{z}(\mathrm{ESI})(\mathrm{M}+\mathrm{Na})^{+} \mathrm{C}_{15} \mathrm{H}_{18} \mathrm{NaO}_{7}\right.$ requires 333.0945, found 333.0943]. 
Synthesis of the 6-O-(p-methoxybenzyl)-2,4-(di-O-(1R,4R)-1,4-diphenylbutanephospho) myoinositol orthoformate 11a and 6-O-(p-methoxybenzyl)-2,4-(di-O-(1R,4R)-1,4-

diphenylbutanephospho) myo-inositol orthoformate 11b
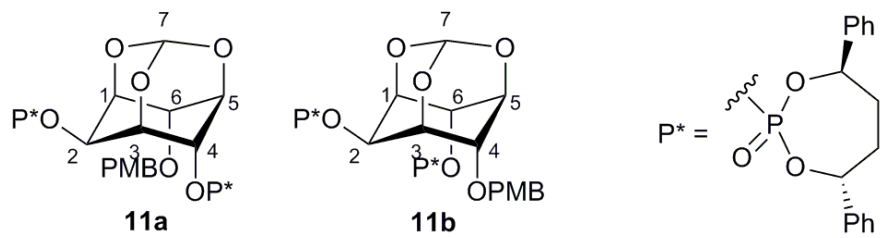

Synthesized from ( \pm )- 6 following procedure A. The obtained residue was purified by flash chromatography $\left(\mathrm{SiO}_{2}\right.$, EtOAc/hexane 30 -> 50\%); 11a was obtained in ratio 10:1 and was repurified by $\mathrm{FC}\left(\mathrm{SiO}_{2}\right.$, EtOAc/toluene $\left.10->20 \%\right)$ to obtain pure $11 \mathrm{a}^{[\mathrm{cc}]}$ as a white solid ( $\left.\mathrm{dr}>99: 1,24 \%{ }^{\left[{ }^{[a]}\right.}\right) .11 \mathbf{b}^{[\mathrm{a}]}$ was obtained in a ratio $14: 1$, and was repurified by $\mathrm{FC}\left(\mathrm{SiO}_{2}\right.$, EtOAc/toluene $\left.10->20 \%\right)$ to obtain pure $11 \mathbf{b}^{[\mathrm{cc}]}$ as a white solid $\left(\mathrm{dr}>99: 1,16 \%^{[\mathrm{la}]}\right)$.

[a] Yield based on the diastereomer itself, which represent $50 \%$ of the racemic starting material.

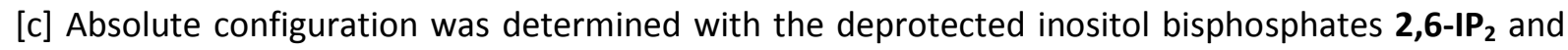
2,4-IP .

11a ${ }^{[c]}:[\alpha]_{D}^{23}+29.0\left(c\right.$ 0.50, $\left.\mathrm{CHCl}_{3}\right) ;{ }^{1} \mathrm{H}$ NMR $\left(400 \mathrm{MHz}, \mathrm{CDCl}_{3}\right) \delta$ (ppm) 7.42-7.47 (4H, m, Ph), 7.15-7.39 $(16 \mathrm{H}, \mathrm{m}, \mathrm{Ph}), 6.97(2 \mathrm{H}, \mathrm{d}, J 8.6 \mathrm{~Hz}, \mathrm{Ph}), 6.67(2 \mathrm{H}, \mathrm{d}, J 8.6 \mathrm{~Hz}, \mathrm{Ph}), 5.66(1 \mathrm{H}, \mathrm{dd}, J$ 9.4, $7.4 \mathrm{~Hz}, \mathrm{CH}-\mathrm{Ph})$, 5.45-5.54 (2H, m, CH-Ph), $5.43\left(1 \mathrm{H}, \mathrm{d},{ }^{5} J_{\mathrm{HH}} 1.1 \mathrm{~Hz}, \mathrm{H}-\mathrm{C} 7\right), 5.28(1 \mathrm{H}, \mathrm{dd}, J$ 9.4, $7.4 \mathrm{~Hz}, \mathrm{CH}-\mathrm{Ph}), 5.20(1 \mathrm{H}$, $\left.\mathrm{dt},{ }^{3} J_{\mathrm{HP}} 7.4,{ }^{3} J_{\mathrm{HH}} 3.5 \mathrm{~Hz}, \mathrm{H}-\mathrm{C} 6\right), 5.14\left(1 \mathrm{H}, \mathrm{dd},{ }^{3} \mathrm{~J}_{\mathrm{HP}} 7.2,{ }^{5} \mathrm{~J}_{\mathrm{HH}} 1.1 \mathrm{~Hz}, \mathrm{H}-\mathrm{C} 2\right), 4.58(1 \mathrm{H}, \mathrm{br}, \mathrm{H}-\mathrm{C} 1), 4.28(1 \mathrm{H}, \mathrm{br}$, $\mathrm{H}-\mathrm{C} 5), 4.23(1 \mathrm{H}, \mathrm{br}, \mathrm{H}-\mathrm{C} 3), 4.19\left(1 \mathrm{H}, \mathrm{d},{ }^{2} J_{\mathrm{HH}} 11.5, \mathrm{CH}_{2}-\mathrm{Ph}\right), 4.11\left(1 \mathrm{H}, \mathrm{d},{ }^{2} \mathrm{~J}_{\mathrm{HH}} 11.5, \mathrm{CH}_{2}-\mathrm{Ph}\right), 4.08(1 \mathrm{H}, \mathrm{br}$, H-C4), $3.72\left(3 \mathrm{H}, \mathrm{s}, \mathrm{O}-\mathrm{CH}_{3}\right), 2.35-2.42\left(2 \mathrm{H}, \mathrm{m}, \mathrm{CH}_{2}\right), 2.13-2.27\left(4 \mathrm{H}, \mathrm{m}, \mathrm{CH}_{2}\right), 1.95-2.01\left(2 \mathrm{H}, \mathrm{m}, \mathrm{CH}_{2}\right)$; ${ }^{13} \mathrm{C}$ NMR $\left(101 \mathrm{MHz}, \mathrm{CDCl}_{3}\right) \delta(\mathrm{ppm}) 159.5(\mathrm{iPh}), 140.6\left(\mathrm{~d},{ }^{3} J_{\mathrm{CP}} 10.4 \mathrm{~Hz}, 2 \mathrm{xiPh}\right), 140.3\left(\mathrm{~d},{ }^{3} J_{\mathrm{CP}} 9.9 \mathrm{~Hz}, i \mathrm{Ph}\right)$, 140.1 (d, $\left.{ }^{3} J_{\mathrm{CP}} 9.9 \mathrm{~Hz}, \mathrm{Ph}\right), 129.7$ (2xPh), 129.1 (iPh), 128.77 (2xPh), 128.76 (2xPh), 128.72 (2xPh), $128.68(2 x \mathrm{Ph}), 128.53(\mathrm{Ph}), 128.50(\mathrm{Ph}), 128.45(\mathrm{Ph}), 128.34(\mathrm{Ph}), 125.93(4 \times \mathrm{Ph}), 125.85(2 \times \mathrm{Ph}), 125.8$ (2xPh), 113.9 (2xPh), 102.9 (C7), 81.2 (d, ${ }^{2} \mathrm{~J}_{\mathrm{CP}} 6.1 \mathrm{~Hz}, \mathrm{CH}-\mathrm{Ph}$ ), 80.8 (d, ${ }^{2} J_{\mathrm{CP}} 6.2 \mathrm{~Hz}, \mathrm{CH}-\mathrm{Ph}$ ), 80.2 (d, ${ }^{2} J_{\mathrm{CP}} 3.6 \mathrm{~Hz}, \mathrm{CH}-\mathrm{Ph}$ ), 79.9 (d, $\left.{ }^{2} J_{\mathrm{CP}} 2.6 \mathrm{~Hz}, \mathrm{CH}-\mathrm{Ph}\right), 72.1$ (C4), $71.2\left(\mathrm{t},{ }^{3} \mathrm{~J}_{\mathrm{CP}} 5.4 \mathrm{~Hz}, \mathrm{C} 1\right), 70.9$ (d, ${ }^{2} J_{\mathrm{CP}} 4.8 \mathrm{~Hz}$, C6), $70.8\left(\mathrm{CH}_{2}-\mathrm{Ph}\right), 70.5\left(\mathrm{~d},{ }^{3} J_{\mathrm{CP}} 3.0 \mathrm{~Hz}, \mathrm{C} 3\right), 68.0\left(\mathrm{~d},{ }^{3} \mathrm{~J}_{\mathrm{CP}} 5.0 \mathrm{~Hz}, \mathrm{C} 5\right), 67.1$ (d, $\left.{ }^{2} \mathrm{JP}_{\mathrm{CP}} 3.3 \mathrm{~Hz}, \mathrm{C} 2\right), 55.4$ (O$\left.\mathrm{CH}_{3}\right), 37.6\left(\mathrm{CH}_{2}\right), 37.5\left(\mathrm{CH}_{2}\right), 37.3\left(\mathrm{CH}_{2}\right), 36.9\left(\mathrm{CH}_{2}\right) ;{ }^{31} \mathrm{P}$ NMR (162 MHz, ${ }^{1} \mathrm{H}$-decoupled, $\left.\mathrm{CDCl}_{3}\right) \delta(\mathrm{ppm})$ $0.82(1 \mathrm{P}),-1.84(1 \mathrm{P}) ;\left[\mathrm{m} / \mathbf{z}(\mathrm{ESI})(\mathrm{M}+\mathrm{H})^{+} \mathrm{C}_{47} \mathrm{H}_{49} \mathrm{O}_{13} \mathrm{P}_{2}\right.$ requires 883.2643, found 883.2620].

11b ${ }^{[\mathrm{cc}]}:[\boldsymbol{\alpha}]_{\boldsymbol{D}}^{25}+43.0\left(\right.$ c 0.35, $\left.\mathrm{CHCl}_{3}\right) ;{ }^{1} \mathrm{H}$ NMR $\left(400 \mathrm{MHz}, \mathrm{CDCl}_{3}\right) \delta(\mathrm{ppm}) 7.43(2 \mathrm{H}, \mathrm{d}, J 6.9 \mathrm{~Hz}, \mathrm{Ph}), 7.24-$ $7.39(18 \mathrm{H}, \mathrm{m}, \mathrm{Ph}), 7.16(2 \mathrm{H}, \mathrm{d}, J 8.6 \mathrm{~Hz}, \mathrm{Ph}), 6.69(2 \mathrm{H}, \mathrm{d}, J 8.6 \mathrm{~Hz}, \mathrm{Ph}), 5.64(1 \mathrm{H}, \mathrm{dd}, J$ 9.8, $7.4 \mathrm{~Hz}$, $\mathrm{CH}-\mathrm{Ph}), 5.52(1 \mathrm{H}, \mathrm{dd}, J$ 9.9, $7.4 \mathrm{~Hz}, \mathrm{CH}-\mathrm{Ph}), 5.48(1 \mathrm{H}, \mathrm{dd}, \mathrm{J}$ 10.0, $7.4 \mathrm{~Hz}, \mathrm{CH}-\mathrm{Ph}), 5.44\left(1 \mathrm{H}, \mathrm{d},{ }^{5} \mathrm{JHH}_{\mathrm{HH}} 0.5 \mathrm{~Hz}\right.$, 
$\mathrm{H}-\mathrm{C} 7)$, 5.07-5.15 (3H, m, CH-Ph, H-C2, H-C4), $4.58(1 \mathrm{H}, \mathrm{br}, \mathrm{H}-\mathrm{C} 5), 4.54\left(1 \mathrm{H}, \mathrm{d},{ }^{2} J_{\mathrm{HH}} 11.3, \mathrm{CH}_{2}-\mathrm{Ph}\right)$, 4.50-4.52 (1H, m, H-C1), $4.46\left(1 \mathrm{H}, \mathrm{d}^{2}{ }^{2} \mathrm{JH}_{\mathrm{HH}} 11.3, \mathrm{CH}_{2}-\mathrm{Ph}\right), 4.33(1 \mathrm{H}, \mathrm{br}, \mathrm{H}-\mathrm{C} 6), 4.15$ (1H, br, H-C3), 3.71 $\left(3 \mathrm{H}, \mathrm{s}, \mathrm{O}-\mathrm{CH}_{3}\right), 1.92-2.44\left(8 \mathrm{H}, \mathrm{m}, \mathrm{CH}_{2}\right) ;{ }^{13} \mathrm{C}$ NMR $\left(126 \mathrm{MHz}, \mathrm{CDCl}_{3}\right) \delta(\mathrm{ppm}) 159.4(\mathrm{Ph}), 140.4(\mathrm{~d}$, ${ }^{3} J_{\mathrm{CP}} 4.6 \mathrm{~Hz}, i \mathrm{Ph}$ ), $140.3\left(\mathrm{~d},{ }^{3} J_{\mathrm{CP}} 4.2 \mathrm{~Hz}, i \mathrm{Ph}\right), 140.1\left(\mathrm{~d},{ }^{3} \mathrm{JP}_{\mathrm{CP}} 10.2 \mathrm{~Hz}, i \mathrm{Ph}\right), 140.0\left(\mathrm{~d},{ }^{3} J_{\mathrm{CP}} 10.3 \mathrm{~Hz}, i \mathrm{Ph}\right), 129.6$ (2xPh), 129.3 (iPh), 128.8 (2xPh), 128.69 (4xPh), 128.68 (2xPh), $128.6(\mathrm{Ph}), 128.5(\mathrm{Ph}), 128.40(\mathrm{Ph})$, 128.39 (Ph), 126.1 (2xPh), 125.84 (2xPh), 125.76 (2xPh), 125.7 (2xPh), 113.9 (2xPh), 102.9 (C7), 81.0 (d, $\left.{ }^{2} J_{\mathrm{CP}} 6.3 \mathrm{~Hz}, \mathrm{CH}-\mathrm{Ph}\right), 80.9$ (d, ${ }^{2} \mathrm{JP}_{\mathrm{CP}} 6.1 \mathrm{~Hz}, \mathrm{CH}-\mathrm{Ph}$ ), 79.8 (d, $\left.{ }^{2} J_{\mathrm{CP}} 2.9 \mathrm{~Hz}, \mathrm{CH}-\mathrm{Ph}\right), 79.6$ (d, ${ }^{2} \mathrm{JP}_{\mathrm{CP}} 3.1 \mathrm{~Hz}, \mathrm{CH}-$ $\mathrm{Ph}$ ), $72.8(\mathrm{C} 6), 71.3\left(\mathrm{CH}_{2}-\mathrm{Ph}\right), 70.94$ (dd, $\left.{ }^{3} J_{\mathrm{CP}} 8.3,5.4 \mathrm{~Hz}, \mathrm{C} 3\right), 70.89\left(\mathrm{~d},{ }^{3} \mathrm{~J}_{\mathrm{CP}} 3.8 \mathrm{~Hz}, \mathrm{C} 1\right), 70.7\left(\mathrm{~d},{ }^{2} J_{\mathrm{CP}} 4.1\right.$ $\mathrm{Hz}, \mathrm{C} 4), 67.7\left(\mathrm{~d},{ }^{3} \mathrm{JP}_{\mathrm{CP}} 1.2 \mathrm{~Hz}, \mathrm{C} 5\right), 67.3\left(\mathrm{~d},{ }^{2} \mathrm{~J}_{\mathrm{CP}} 4.1 \mathrm{~Hz}, \mathrm{C} 2\right), 55.3\left(\mathrm{O}-\mathrm{CH}_{3}\right), 37.5\left(\mathrm{CH}_{2}\right), 37.3\left(\mathrm{CH}_{2}\right), 36.82$ $\left(\mathrm{CH}_{2}\right), 36.76\left(\mathrm{CH}_{2}\right) ;{ }^{31} \mathrm{P}$ NMR (162 MHz, ${ }^{1} \mathrm{H}$-decoupled, $\left.\mathrm{CDCl}_{3}\right) \delta(\mathrm{ppm})-0.90(1 \mathrm{P}),-1.32$ (1P); [ $\mathrm{m} / \mathbf{z}$ (ESI) $(\mathrm{M}+\mathrm{H})^{+} \mathrm{C}_{47} \mathrm{H}_{49} \mathrm{O}_{13} \mathrm{P}_{2}$ requires 883.2643, found 883.2631].

\section{Synthesis of 2,4-di-O-phosphate myo-inositol 2,4-IP ${ }_{2}$ and 2,6-di-O-phosphate myo-inositol 2,6-IP}

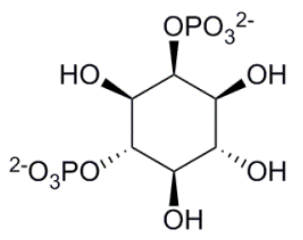

2,4-IP

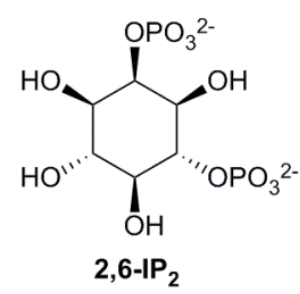

2,6-IP 2

Synthesized from $\mathbf{1 1 a}$ and $\mathbf{1 1 b}$ according to procedure B with a reaction time of 1.5 h. $\mathbf{2 , 4}-\mathbf{I P}_{\mathbf{2}}$ was obtained as a white solid (3.9 $\mathrm{mg}, 95 \%) ; \mathbf{2 , 6 - I \mathbf { P } _ { 2 }}$ was obtained as a white solid (3.7 $\mathrm{mg}, \mathbf{8 7 \%}$ ); respectively.

${ }^{1} \mathrm{H}$ NMR (400 MHz, MeOD) $\delta(\mathrm{ppm}) 4.70\left(1 \mathrm{H}, \mathrm{dt},{ }^{3} \mathrm{~J}_{\mathrm{HP}} 8.2, \mathrm{~J} 2.0 \mathrm{~Hz}, \mathrm{H}-\mathrm{C} 2\right), 4.32\left(1 \mathrm{H}, \mathrm{q},{ }^{3} J_{\mathrm{HP}} 9.2, J 9.2 \mathrm{~Hz}\right.$, H-C6), $3.74(1 \mathrm{H}, \mathrm{d}, J 9.4 \mathrm{~Hz}, \mathrm{H}-\mathrm{C} 1), 3.67(1 \mathrm{H}, \mathrm{t}, J 9.5 \mathrm{~Hz}, \mathrm{H}-\mathrm{C} 4), 3.55$ (1H, dt, J 9.9, $2.0 \mathrm{~Hz}, \mathrm{H}-\mathrm{C} 3), 3.43$ $(1 \mathrm{H}, \mathrm{t}, J 9.1 \mathrm{~Hz}, \mathrm{H}-\mathrm{C} 5) ;{ }^{13} \mathrm{C}$ NMR $(101 \mathrm{MHz}, \mathrm{MeOD}) \delta(\mathrm{ppm}) 81.3\left(\mathrm{~d},{ }^{2} J_{\mathrm{CP}} 6.3 \mathrm{~Hz}, \mathrm{C} 2\right), 81.1\left(\mathrm{~d},{ }^{2} J_{\mathrm{CP}} 6.2 \mathrm{~Hz}\right.$, C6), $75.3\left(\mathrm{~d},{ }^{3} J_{\mathrm{CP}} 3.6 \mathrm{~Hz}, \mathrm{C} 5\right), 73.9(\mathrm{C} 4), 71.9$ (d, $\left.{ }^{3} J_{\mathrm{CP}} 3.3 \mathrm{~Hz}, \mathrm{C} 3\right), 71.2\left(\mathrm{t},{ }^{3} \mathrm{JP}_{\mathrm{CP}} 3.3 \mathrm{~Hz}, \mathrm{C} 1\right) ;{ }^{31} \mathbf{P}$ NMR (162 MHz, ${ }^{1} \mathrm{H}$-decoupled, MeOD) $\delta$ (ppm) 1.10 (1P), 0.74 (1P); [m/z (ESI) (M+H) ${ }^{+} \mathrm{C}_{6} \mathrm{H}_{15} \mathrm{O}_{12} \mathrm{P}_{2}$ requires 341.0033, measured 341.0034].

Absolute configuration was determined by optical rotation measurement that was compared with literature. ${ }^{6}$ In order to ensure the comparison with the literature, the samples were prepared in

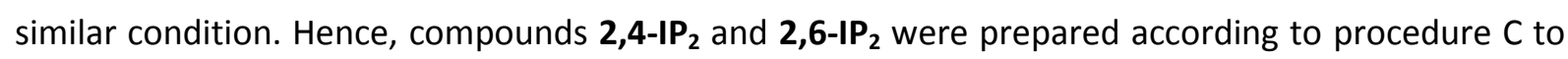
obtain the lithium salt.

2,4-IP $:[\alpha]_{D}^{23}+10.78\left(c 0.117, \mathrm{H}_{2} \mathrm{O}\right.$, lithium salt, $\mathrm{pH}=7$ );

2,6-IP $:[\alpha]_{D}^{23}-7.43\left(c 0.183, \mathrm{H}_{2} \mathrm{O}\right.$, lithium salt, $\left.\mathrm{pH}=7\right)\left\{\text { lit. }[\alpha]_{D}^{22}-4.3\left(c 0.7, \mathrm{H}_{2} \mathrm{O}, \mathrm{pH}=10\right)\right\}^{6}$ 


\subsection{Synthesis of 1,5-diphosphate myo-inositol 1,5-IP}

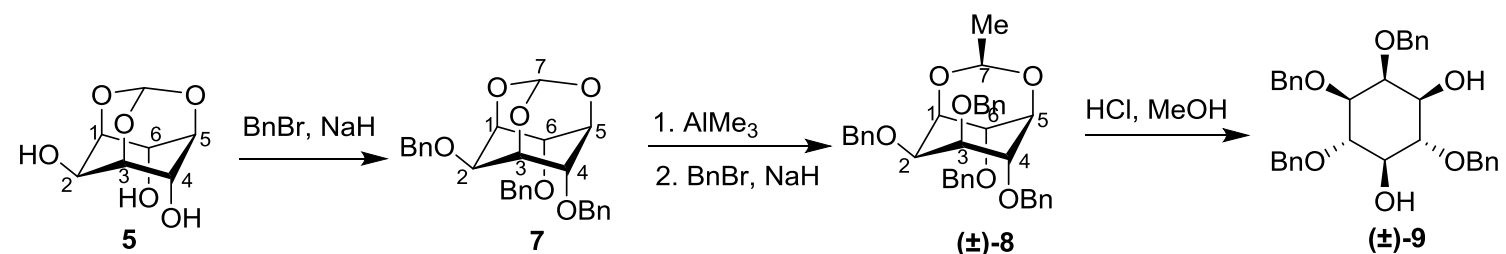

(士)-8

$( \pm)-9$

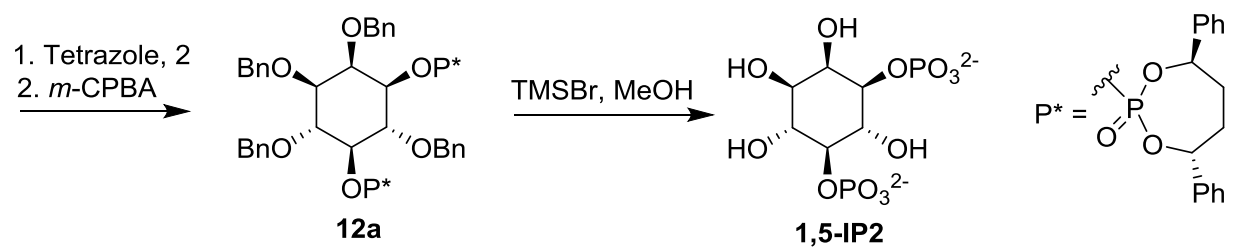

\section{Synthesis of ( \pm )-2,3,4,6-tetra-O-benzyl-1,5-O-ethyldiene myo-inositol ( \pm )-8}

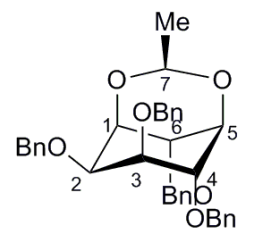

$( \pm)-8$

To a solution of ( \pm )-2,4,6-tri-O-benzyl-1,5-O-ethyldiene myo-inositol (483 mg, $1.01 \mathrm{mmol}, 1$ equiv) in DMF dry $(10.1 \mathrm{~mL}, 0.1 \mathrm{M})$ at $0{ }^{\circ} \mathrm{C}$ was added portionwise $\mathrm{NaH}(60 \%$ in oil w/w; $57 \mathrm{mg}, 1.42 \mathrm{mmol}$, 1.4 equiv). The reaction mixture was then treated with benzyl chloride ( $217 \mu \mathrm{L}, 1.72 \mathrm{mmol}, 1.7$ equiv) and stirred for $20 \mathrm{~h}$. The mixture was neutralized with $\mathrm{MeOH}$ and concentrated in vacuo at $60^{\circ} \mathrm{C}$. The crude was diluted with EtOAc and washed with $\mathrm{H}_{2} \mathrm{O}$ and then with brine. The organic phase was dried over $\mathrm{Na}_{2} \mathrm{SO}_{4}$ filtered and concentrated in vacuo. Purification by flash column chromatography $\left(\mathrm{SiO}_{2}\right.$, EtOAc/hexane 10\%) afforded ( \pm )-8 as a white solid (528 mg, $0.93 \mathrm{mmol}, 92 \%)$.

${ }^{1} \mathrm{H}$ NMR $\left(400 \mathrm{MHz}, \mathrm{CDCl}_{3}\right) \delta(\mathrm{ppm}) 7.20-7.38(20 \mathrm{H}, \mathrm{m}, \mathrm{Ph}), 5.38(1 \mathrm{H}, \mathrm{q}, J 4.8 \mathrm{~Hz}, \mathrm{H}-\mathrm{C} 7), 4.78(1 \mathrm{H}, \mathrm{d}$, J $\left.11.7 \mathrm{~Hz}, \mathrm{CH}_{2}-\mathrm{Ph}\right), 4.77\left(1 \mathrm{H}, \mathrm{d}, J 11.9 \mathrm{~Hz}, \mathrm{CH}_{2}-\mathrm{Ph}\right), 4.73\left(1 \mathrm{H}, \mathrm{d}, J 11.9 \mathrm{~Hz}, \mathrm{CH}_{2}-\mathrm{Ph}\right), 4.68(1 \mathrm{H}, \mathrm{d}$, J $\left.11.4 \mathrm{~Hz}, \mathrm{CH}_{2}-\mathrm{Ph}\right), 4.65\left(1 \mathrm{H}, \mathrm{d}, J 11.6 \mathrm{~Hz}, \mathrm{CH}_{2}-\mathrm{Ph}\right), 4.54\left(1 \mathrm{H}, \mathrm{d}, J 11.7 \mathrm{~Hz}, \mathrm{CH}_{2}-\mathrm{Ph}\right), 4.48(1 \mathrm{H}, \mathrm{d}$, J $\left.11.7 \mathrm{~Hz}, \mathrm{CH}_{2}-\mathrm{Ph}\right), 4.42-4.45(1 \mathrm{H}, \mathrm{m}), 4.41\left(1 \mathrm{H}, \mathrm{d}, J 11.5 \mathrm{~Hz}, \mathrm{CH}_{2}-\mathrm{Ph}\right), 4.35(1 \mathrm{H}, \mathrm{dd}, J 3.6,1.9 \mathrm{~Hz}), 4.30$ $(1 \mathrm{H}, \mathrm{t}, J 7.9 \mathrm{~Hz}), 4.12(1 \mathrm{H}, \mathrm{t}, J 7.5 \mathrm{~Hz}), 4.06(1 \mathrm{H}, \mathrm{d}, J 8.0 \mathrm{~Hz}), 3.97(1 \mathrm{H}, \mathrm{td}, J 4.0,0.9 \mathrm{~Hz}), 1.25(3 \mathrm{H}, \mathrm{d}$, J $4.9 \mathrm{~Hz}$ ); ${ }^{13} \mathrm{C}$ NMR (126 MHz, CDCl ${ }_{3}$ ) (ppm) 138.5 (iPh), 138.4 (iPh), 137.9 (iPh), 137.6 (iPh), 128.50 (2xPh), 128.47 (2xPh), 128.4 (2xPh), 128.3 (2xPh), 128.2 (2xPh), 128.1 (2xPh), $127.92(\mathrm{Ph}), 127.90$ (2xPh), 127.58 (Ph), 127.6 (2C, Ph), 127.52 (Ph), 127.47 (Ph), 90.9 (C7), 80.9, 76.3, 73.4, 73.3, 72.4 $\left(\mathrm{CH}_{2}-\mathrm{Ph}\right), 72.32\left(\mathrm{CH}_{2}-\mathrm{Ph}\right), 72.26\left(\mathrm{CH}_{2}-\mathrm{Ph}\right), 71.3\left(\mathrm{CH}_{2}-\mathrm{Ph}\right), 69.8,68.6,20.8\left(\mathrm{CH}_{3}\right) ;\left[\mathrm{m} / \mathbf{z}(\mathrm{ESI})(\mathrm{M}+\mathrm{Na})^{+}\right.$ $\mathrm{C}_{36} \mathrm{H}_{38} \mathrm{NaO}_{6}$ requires 589.2561, found 589.2556]. 
Synthesis of ( \pm )-2,3,4,6-tetra-O-benzyl myo-inositol ( \pm )-9

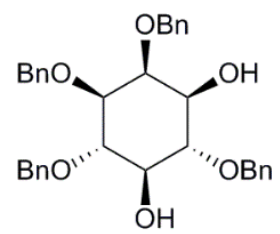

$( \pm)-9$

A solution of ( \pm ) $-8\left(60.9 \mathrm{mg}, 107 \mu \mathrm{mol}, 1\right.$ equiv) in $\mathrm{MeOH}(6.5 \mathrm{~mL}, 0.02 \mathrm{M})$ at $0{ }^{\circ} \mathrm{C}$ was treated with $\mathrm{HCl}_{\mathrm{cc}}(75 \mu \mathrm{L}, 900 \mu \mathrm{mol}, 8.4$ equiv) and stirred at $\mathrm{rt}$ for $2.5 \mathrm{~h}$. The mixture was concentrated in vacuo at $40{ }^{\circ} \mathrm{C}$ and co-evaporated with toluene. Purification by flash column chromatography $\left(\mathrm{SiO}_{2}\right.$, EtOAc/hexane 30\%) afforded ( \pm )-9 as a white solid (52.6 mg, $97 \mu \mathrm{mol}, 91 \%)$.

${ }^{1} \mathrm{H}$ NMR $\left(400 \mathrm{MHz}, \mathrm{CDCl}_{3}\right) \delta(\mathrm{ppm})$ 7.28-7.41 $(20 \mathrm{H}, \mathrm{m}, \mathrm{Ph}), 5.01\left(1 \mathrm{H}, \mathrm{d}, J 11.6 \mathrm{~Hz}, \mathrm{CH}_{2}-\mathrm{Ph}\right), 5.01(1 \mathrm{H}, \mathrm{d}$, J $\left.11.2 \mathrm{~Hz}, \mathrm{CH}_{2}-\mathrm{Ph}\right), 4.92\left(1 \mathrm{H}, \mathrm{d}, J 11.3 \mathrm{~Hz}, \mathrm{CH}_{2}-\mathrm{Ph}\right), 4.81\left(1 \mathrm{H}, \mathrm{d}, J 11.3 \mathrm{~Hz}, \mathrm{CH}_{2}-\mathrm{Ph}\right), 4.79(1 \mathrm{H}, \mathrm{d}$, J $\left.11.2 \mathrm{~Hz}, \mathrm{CH}_{2}-\mathrm{Ph}\right), 4.76\left(1 \mathrm{H}, \mathrm{d}, J 11.6 \mathrm{~Hz}, \mathrm{CH}_{2}-\mathrm{Ph}\right), 4.70\left(2 \mathrm{H}, \mathrm{s}, \mathrm{CH}_{2}-\mathrm{Ph}\right), 4.08(1 \mathrm{H}, \mathrm{t}, J 2.5 \mathrm{~Hz}, \mathrm{H}-\mathrm{C} 2)$, $3.93(1 \mathrm{H}, \mathrm{t}, J 9.4 \mathrm{~Hz}, \mathrm{H}-\mathrm{C} 4), 3.72(1 \mathrm{H}, \mathrm{t}, J 9.3 \mathrm{~Hz}, \mathrm{H}-\mathrm{C} 6), 3.55$ (1H, td, J 9.2, $2.0 \mathrm{~Hz}, \mathrm{H}-\mathrm{C} 5), 3.48-3.55(1 \mathrm{H}$, m, H-C1), $3.47(1 \mathrm{H}, \mathrm{dd}, J$ 9.8, $2.4 \mathrm{~Hz}, \mathrm{H}-\mathrm{C} 3), 2.54(1 \mathrm{H}, \mathrm{d}, J 2.1 \mathrm{~Hz}, \mathrm{OH}-\mathrm{C} 6), 2.34(1 \mathrm{H}, \mathrm{d}, J 6.3 \mathrm{~Hz}, \mathrm{OH}-\mathrm{C} 1)$; ${ }^{13} \mathrm{C}$ NMR $\left(126 \mathrm{MHz}, \mathrm{CDCl}_{3}\right) \delta$ (ppm) 138.83 (iPh), 138.82 (iPh), 138.81 (iPh), 138.23 (iPh), 128.64 (3xPh), 128.59 (2xPh), 128.5 (2xPh), 128.2 (2xPh), 128.1 (2xPh), 127.91 (3xPh), 127.90 (2xPh), 127.88 (Ph), 127.8 (Ph), 127.7 (2xPh), 81.8 (C6), 81.4 (C4), 81.0 (C3), 77.2 (C2),75.6 ( $\left.\mathrm{CH}_{2}-\mathrm{Ph}\right), 75.2$ (C5), 75.1 $\left(\mathrm{CH}_{2}-\mathrm{Ph}\right), 74.9\left(\mathrm{CH}_{2}-\mathrm{Ph}\right), 72.9\left(\mathrm{CH}_{2}-\mathrm{Ph}\right), 72.3(\mathrm{C} 1) ;\left[\mathrm{m} / \mathbf{z}(\mathrm{ESI})(\mathrm{M}+\mathrm{Na})^{+} \mathrm{C}_{34} \mathrm{H}_{36} \mathrm{NaO}_{6}\right.$ requires 563.2404, found 563.2405].

Synthesis of the 2,3,4,6-tetra-O-benzyl-1,5-(di-O-(1R,4R)-1,4-diphenylbutanephospho) myo-inositol 12a and the 1,2,4,6-tetra-O-benzyl-3,5-(di-O-(1R,4R)-1,4-diphenylbutanephospho) myo-inositol 12b

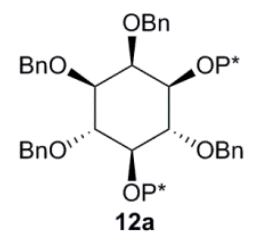

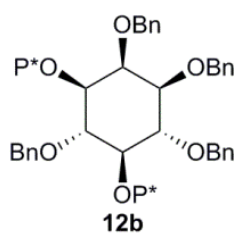<smiles>CC(C)P1(=O)OC(c2ccccc2)CCC(c2ccccc2)O1</smiles>

Synthesized from ( \pm )-9; the obtained residue was purified by flash chromatography $\left(\mathrm{SiO}_{2}, \mathrm{EtOAc} /\right.$ hexane $30->40 \%$ ) affording the diastereomeric mixture $12 \mathrm{a}$ and $\mathbf{1 2 b}$ as white solid (229 $\mathrm{mg}, 98 \%$ ). Crystallization of the mixture by vapor diffusion method $\left(\mathrm{EtOAc} / \mathrm{Et}_{2} \mathrm{O}\right)$ afforded diastereomer 12a $(\mathrm{dr}$ $>99: 1,52 \%{ }^{[a]}$ ). Mother liquor was crystallized in $\mathrm{iPrOH}$, and diastereomer $\mathbf{1 2} \mathbf{b}$ was recovered (dr $\left.85: 15,11^{[\mathrm{a}]}\right)$.

[a] Yield based on the diastereomer itself, which represent $50 \%$ of the racemic starting material. 
12a: $[\alpha]_{D}^{23}+43.1\left(c\right.$ 0.50, $\left.\mathrm{CHCl}_{3}\right) ;{ }^{1} \mathrm{H}$ NMR $\left(400 \mathrm{MHz}, \mathrm{CDCl}_{3}\right) \delta(\mathrm{ppm}) 7.43(2 \mathrm{H}, \mathrm{dd}, J 7.8,1.6 \mathrm{~Hz}, \mathrm{Ph})$, 7.29-7.38 (8H, m, Ph), 7.05-7.26 (28H, m, Ph), $7.01(2 \mathrm{H}, \mathrm{t}, J 7.3 \mathrm{~Hz}, \mathrm{Ph}), 5.59(1 \mathrm{H}, \mathrm{dd}, J$ 10.1, $6.9 \mathrm{~Hz}$, CH-Ph), 5.53 (1H, td, J 7.2, 4.9 Hz, CH-Ph), 5.37 (1H, dd, J 10.2, 7.5 Hz, CH-Ph), 5.14 (1H, dd, J 10.4, 6.9 $\mathrm{Hz}, \mathrm{CH}-\mathrm{Ph}), 4.97\left(1 \mathrm{H}, \mathrm{d}, J\right.$ 10.3, $\left.\mathrm{CH}_{2}-\mathrm{Ph}\right), 4.89\left(1 \mathrm{H}, \mathrm{d}, J\right.$ J1.5, $\left.\mathrm{CH}_{2}-\mathrm{Ph}\right), 4.81\left(1 \mathrm{H}, \mathrm{d}, J\right.$ 11.5, $\left.\mathrm{CH}_{2}-\mathrm{Ph}\right), 4.63$ (1H, d, J 10.3, $\left.\mathrm{CH}_{2}-\mathrm{Ph}\right), 4.51$ (1H, d, J 10.8, $\left.\mathrm{CH}_{2}-\mathrm{Ph}\right), 4.44-4.51$ (1H, m, H-C5), 4.44-4.46 (1H, m, H-C2), $4.43\left(1 \mathrm{H}, \mathrm{d}, J 11.3, \mathrm{CH}_{2}-\mathrm{Ph}\right), 4.36\left(1 \mathrm{H}, \mathrm{d}, J\right.$ J 11.3, $\left.\mathrm{CH}_{2}-\mathrm{Ph}\right), 4.25\left(1 \mathrm{H}, \mathrm{d}, J 10.8, \mathrm{CH}_{2}-\mathrm{Ph}\right), 4.08-4.17(2 \mathrm{H}, \mathrm{m}$, $\mathrm{H}-\mathrm{C} 1, \mathrm{H}-\mathrm{C} 6), 3.87(1 \mathrm{H}, \mathrm{t}, J 9.5 \mathrm{~Hz}, \mathrm{H}-\mathrm{C} 4), 3.30(1 \mathrm{H}, \mathrm{dd}, J$ 9.8, $2.3 \mathrm{~Hz}, \mathrm{H}-\mathrm{C} 3) ;{ }^{13} \mathrm{C}$ NMR $\left(101 \mathrm{MHz}, \mathrm{CDCl}_{3}\right) \delta$ (ppm) 140.8 (d, $\left.{ }^{3} J_{\mathrm{CP}} 10.2 \mathrm{~Hz}, i \mathrm{Ph}\right), 140.4$ (d, ${ }^{3} \mathrm{CP}_{\mathrm{CP}} 10.0 \mathrm{~Hz}, i \mathrm{Ph}$ ), 140.3 (d, ${ }^{3} J_{\mathrm{CP}} 9.0 \mathrm{~Hz}, i \mathrm{Ph}$ ), 140.2 (d, $\left.{ }^{3} J_{\mathrm{CP}} 10.2 \mathrm{~Hz}, \mathrm{PPh}\right), 138.2(i \mathrm{Ph}), 138.7(i \mathrm{Ph}) 138.1(i \mathrm{Ph}), 137.9$ (iPh), 128.69 (2xPh), $128.68(2 \times \mathrm{Ph}), 128.49$ (2xPh), 128.45 (3xPh), 128.34 (3xPh), 128.30 (2xPh), 128.28 (2xPh), $128.14(\mathrm{Ph}), 128.11(\mathrm{Ph}), 128.4$ (2xPh), 128.0 (2xPh), 128.91 (2xPh), 127.89 (2xPh), 127.7 (2xPh), $127.64(\mathrm{Ph}), 127.55(\mathrm{Ph}), 127.54$ (2xPh), $127.31(\mathrm{Ph}), 127.0(\mathrm{Ph}), 125.9(4 \mathrm{xPh}), 125.74(2 \times \mathrm{Ph}), 125.6774(2 \times \mathrm{Ph}), 81.32$ (dd, ${ }^{2} \mathrm{JP}_{\mathrm{CP}} 6.5$, ${ }^{4} J_{\mathrm{CP}} 2.6 \mathrm{~Hz}, \mathrm{C5}$ ), 80.71 (d, ${ }^{2} J_{\mathrm{CP}} 6.2 \mathrm{~Hz}, \mathrm{CH}-\mathrm{Ph}$ ), 80.69 (d, ${ }^{2} J_{\mathrm{CP}} 6.2 \mathrm{~Hz}, \mathrm{CH}-\mathrm{Ph}$ ), 80.1 (C3), 79.6 (d, ${ }^{2} J_{\mathrm{CP}} 3.0 \mathrm{~Hz}, \mathrm{CH}-\mathrm{Ph}$ ), 79.3 (d, $\left.{ }^{3} J_{\mathrm{CP}} 2.4 \mathrm{~Hz}, \mathrm{C} 4\right), 79.1\left(\mathrm{~d},{ }^{2} \mathrm{~J}_{\mathrm{CP}} 2.8 \mathrm{~Hz}, \mathrm{CH}-\mathrm{Ph}\right.$ ), 78.6 (dd, ${ }^{3} \mathrm{JP}_{\mathrm{CP}} 9.0,4.2 \mathrm{~Hz}, \mathrm{C} 6$ ), $78.1\left(\mathrm{dd},{ }^{2} J_{\mathrm{CP}} 4.8,{ }^{4} J_{\mathrm{CP}} 1.6, \mathrm{~Hz}, \mathrm{C} 1\right), 76.3(\mathrm{C} 2), 75.5\left(\mathrm{CH}_{2}-\mathrm{Ph}\right), 75.3\left(\mathrm{CH}_{2}-\mathrm{Ph}\right), 74.8\left(\mathrm{CH}_{2}-\mathrm{Ph}\right), 72.8\left(\mathrm{CH}_{2}-\mathrm{Ph}\right)$, $38.1\left(\mathrm{CH}_{2}\right), 37.6\left(\mathrm{CH}_{2}\right), 37.1\left(\mathrm{CH}_{2}\right), 37.0\left(\mathrm{CH}_{2}\right) ;{ }^{31} \mathrm{P}$ NMR (162 MHz, ${ }^{1} \mathrm{H}$-decoupled, $\left.\mathrm{CDCl}_{3}\right) \delta(\mathrm{ppm})$ 1.49(1P), -2.24 (1P); [ $\mathrm{m} / \mathbf{z}(\mathrm{ESI})(\mathrm{M}+\mathrm{H})^{+} \mathrm{C}_{50} \mathrm{H}_{51} \mathrm{O}_{12} \mathrm{P}_{2}$ requires 1113.4102, found 1113.4087].

Synthesis of 1,5-diphosphate myo-inositol 1,5-IP

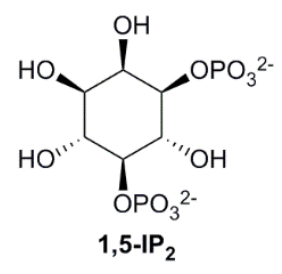

To a solution of $12 \mathrm{a}\left(15.0 \mathrm{mg}, 16.6 \mu \mathrm{mol}, 1\right.$ equiv) dissolved in a minimum amount of $\mathrm{CH}_{2} \mathrm{Cl}_{2}$ was added methanol ( $9 \mathrm{~mL}, 1.8 \mathrm{~mm}$ ) and $\mathrm{Pd}$ on activated carbon $10 \%(\mathrm{w} / \mathrm{w})$ (point of spatula). The reaction mixture was evacuated and flushed with hydrogen gas (operation repeated several times during the reaction time) and was vigorously stirred at $\mathrm{rt}$ for $1.5 \mathrm{~h}$. The reaction mixture was flushed with nitrogen, filtered through a pad of celite and concentrated to dryness. Suspension in acetone, then acetonitrile and chloroform afforded clean product 1,5-IP $\mathbf{P}_{2}$ as a white solid (3.2 $\mathrm{mg}, 89 \%$ ).

1,5-IP ${ }_{2}:[\alpha]_{D}^{23}-4.85\left(c 0.094, \mathrm{H}_{2} \mathrm{O} \text {, lithium salt, } \mathrm{pH}=7\right)^{\mathrm{d}} ;{ }^{1} \mathrm{H}$ NMR $(400 \mathrm{MHz}, \mathrm{MeOD}) \delta(\mathrm{ppm}) 4.20(1 \mathrm{H}$, br, H-C2), 4.03-4.10 (1H, m, H-C1), 3.91-4.02 (2H, m, H-C6, H-C5), 3.81 (1H, t, J 9.0 Hz, H-C4), 3.44 $(1 \mathrm{H}, \mathrm{dd}, J$ 9.7, $2.5 \mathrm{~Hz}, \mathrm{H}-\mathrm{C} 3) ;{ }^{13} \mathrm{C}$ NMR $(101 \mathrm{MHz}, \mathrm{MeOD}) \delta(\mathrm{ppm}) 81.1\left(\mathrm{~d},{ }^{2} J_{\mathrm{CP}} 6.4 \mathrm{~Hz}\right), 77.2\left(\mathrm{~d},{ }^{2} J_{\mathrm{CP}} 5.0\right.$ $\mathrm{Hz}$ ), 71.7 (br), 71.0 (C2), 70.9 (C3), 70.6 (br); ${ }^{31} \mathrm{P}$ NMR (162 MHz, ${ }^{1} \mathrm{H}$-decoupled, MeOD) $\delta$ (ppm) 0.94 (1P), $0.04(1 \mathrm{P}) ;\left[\mathrm{m} / \mathbf{z}(\mathrm{ESI})(\mathrm{M}+\mathrm{H})^{+} \mathrm{C}_{6} \mathrm{H}_{15} \mathrm{O}_{12} \mathrm{P}_{2}\right.$ requires 341.0033, measured 341.0034]. 
[d] 1,5-IP lithium salts were prepared following the procedure $C$ for optical rotation measurement. 


\section{X-Ray Crystallographic Structure}

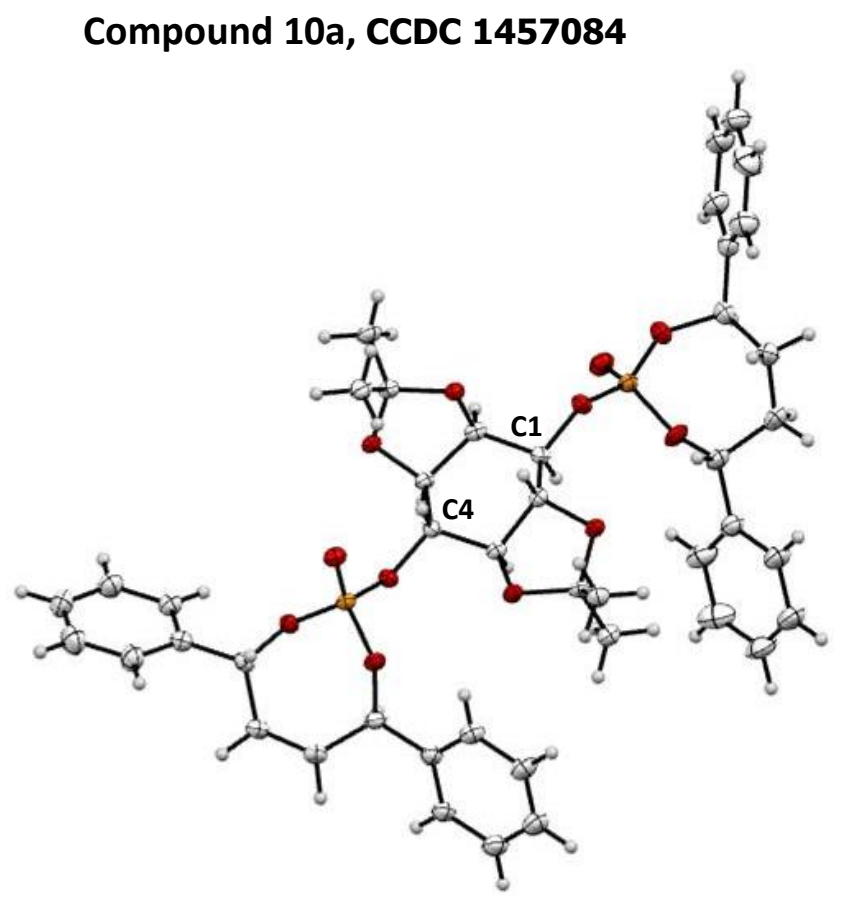

Figure S1 X-Ray structure of compound 10a

Compound 12a, CCDC 1457083

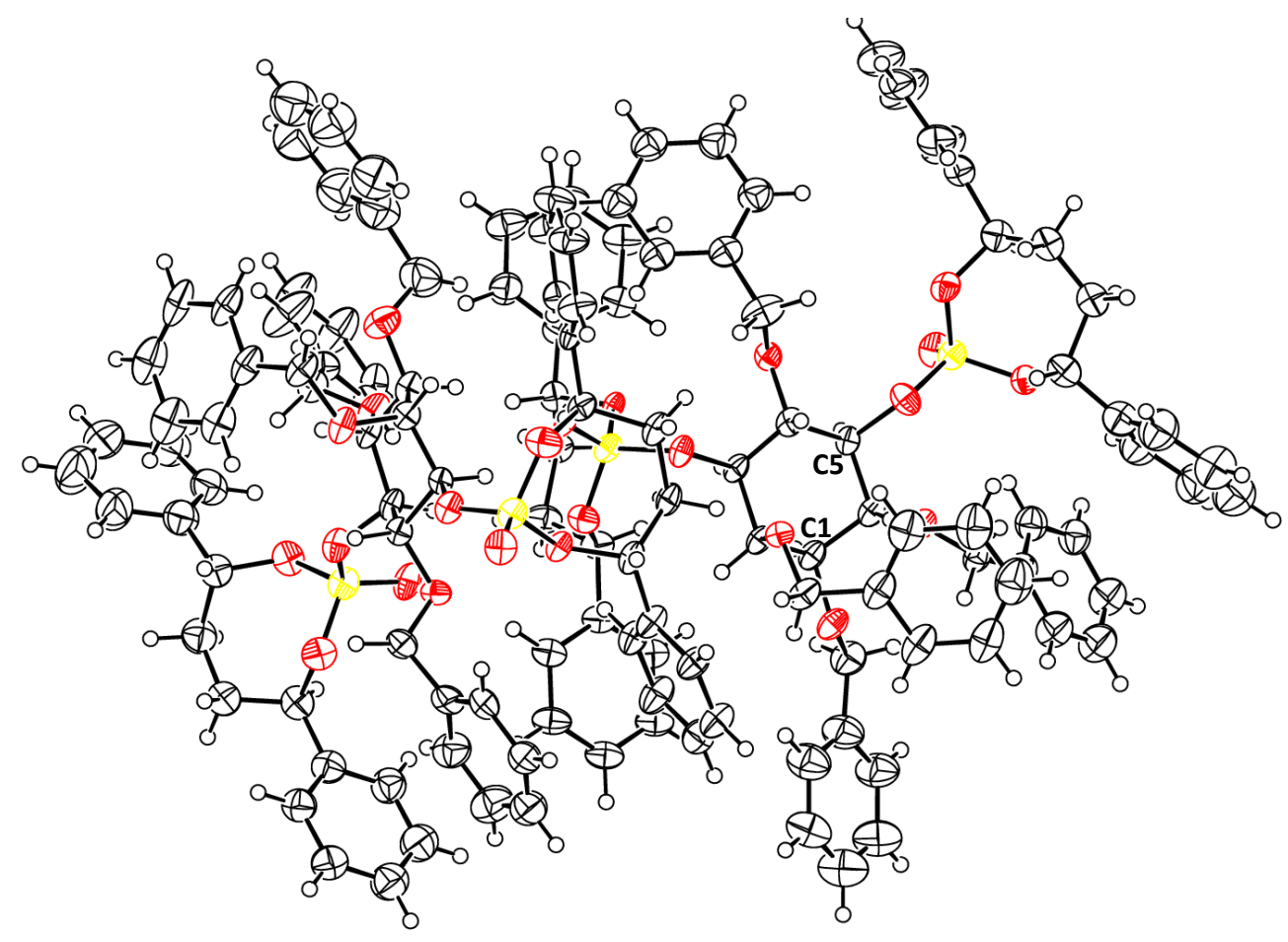

Figure S2 X-Ray structure of compound 12a

The quality of the crystal was poor however it was sufficient for assigning the absolute configuration. 


\section{References}

(1) Jarvis, W. F.; Hoey, M. D.; Finocchio, A. L.; Dittmer, D. C. J. Org. Chem. 1988, 53, 5750-5756.

(2) Kemppainen, E. K.; Sahoo, G.; Valkonen, A.; Pihko, P. M. Org. Lett. 2012, 14, 1086-1089.

(3) Khersonsky, S. M.; Chang, Y.-T. Carbohydr. Res. 2002, 337 (1), 75-78.

(4) Podeschwa, M. A. L.; Plettenburg, O.; Altenbach, H.-J. Eur. J. Org. Chem. 2005, 3101-3115.

(5) Jordan, P. A.; Kayser-Bricker, K. J.; Miller, S. J. Proc. Natl. Acad. Sci. U. S. A. 2010, 107, 2062020624.

(6) Chung, S.-K.; Yu, S.-H.; Chang, Y.-T. J. Carbohydr. Chem. 1998, 17, 385-390. 


\section{NMR spectroscopy spectra}

${ }^{1} \mathrm{H} \mathrm{NMR}, 400 \mathrm{MHz}, \mathrm{CDCl}_{3}$
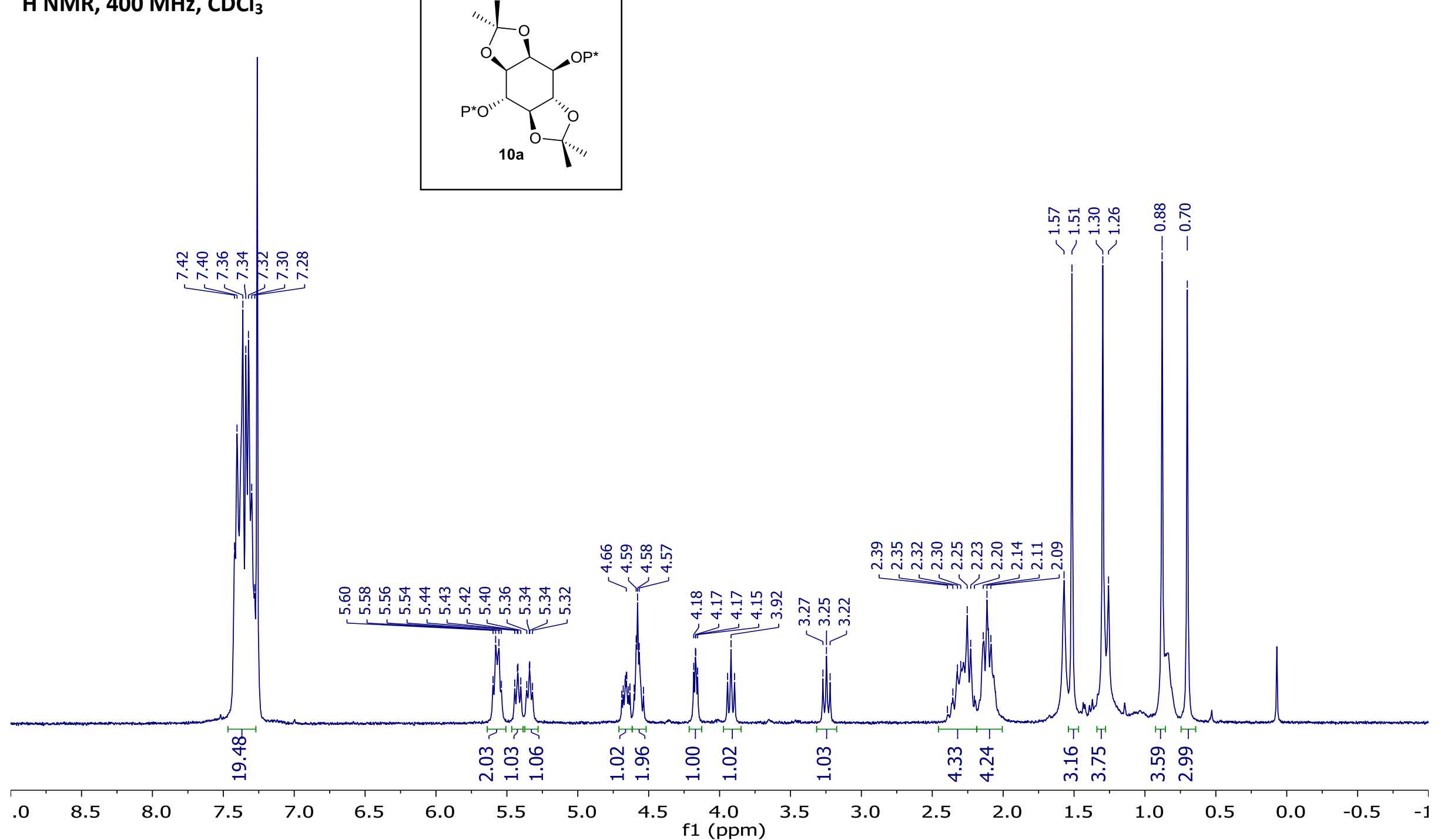
${ }^{13} \mathrm{C}$ NMR, $126 \mathrm{MHz}, \mathrm{CDCl}_{3}$

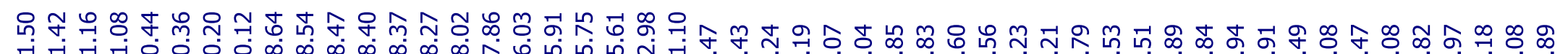

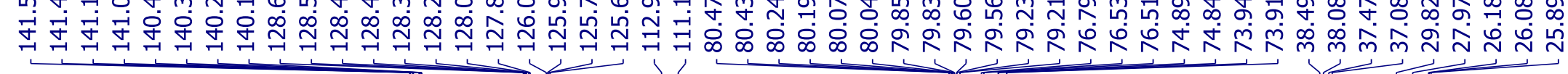
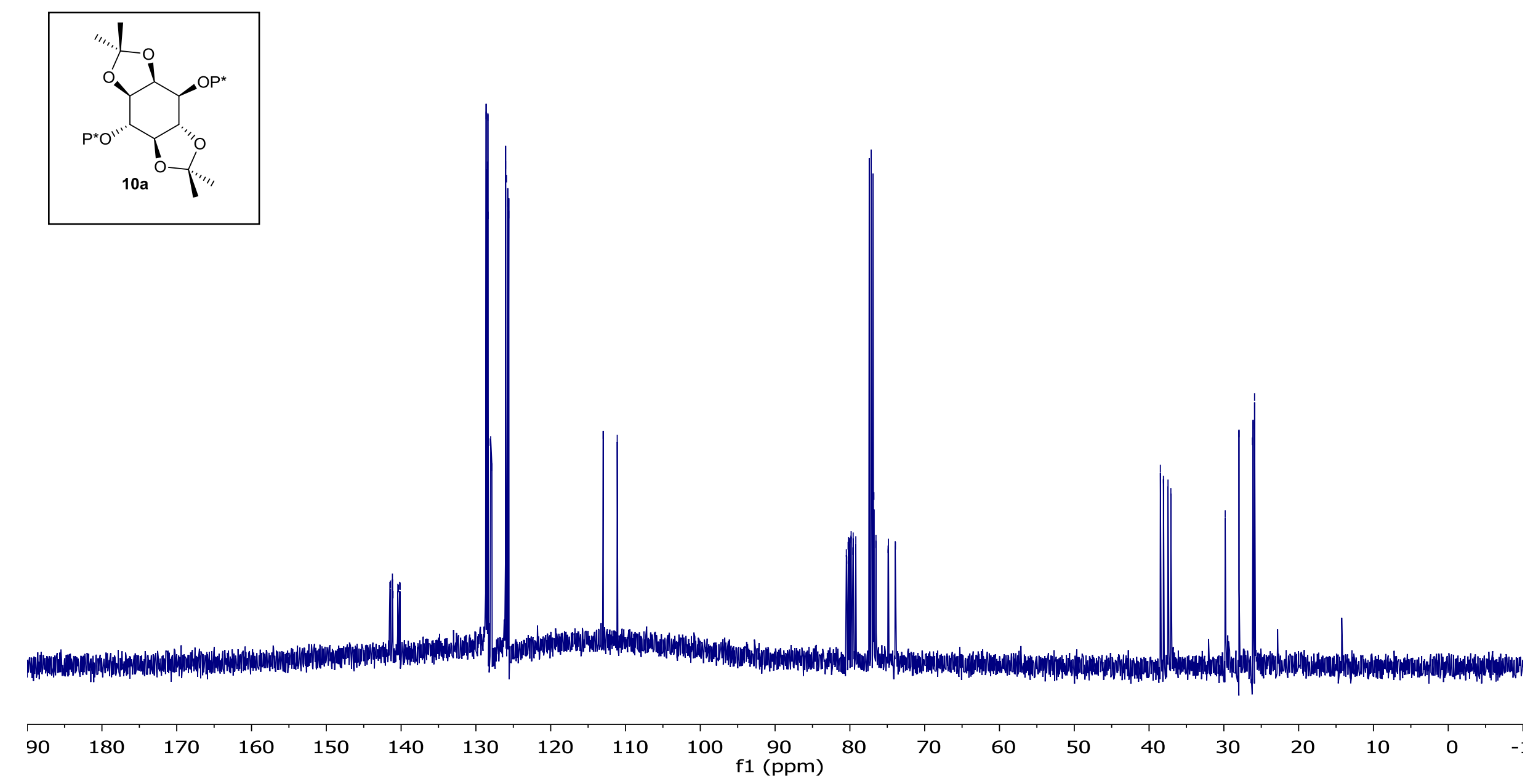
${ }^{31} \mathrm{P}$ NMR, $162 \mathrm{MHz}, \mathrm{CDCl}_{3}$
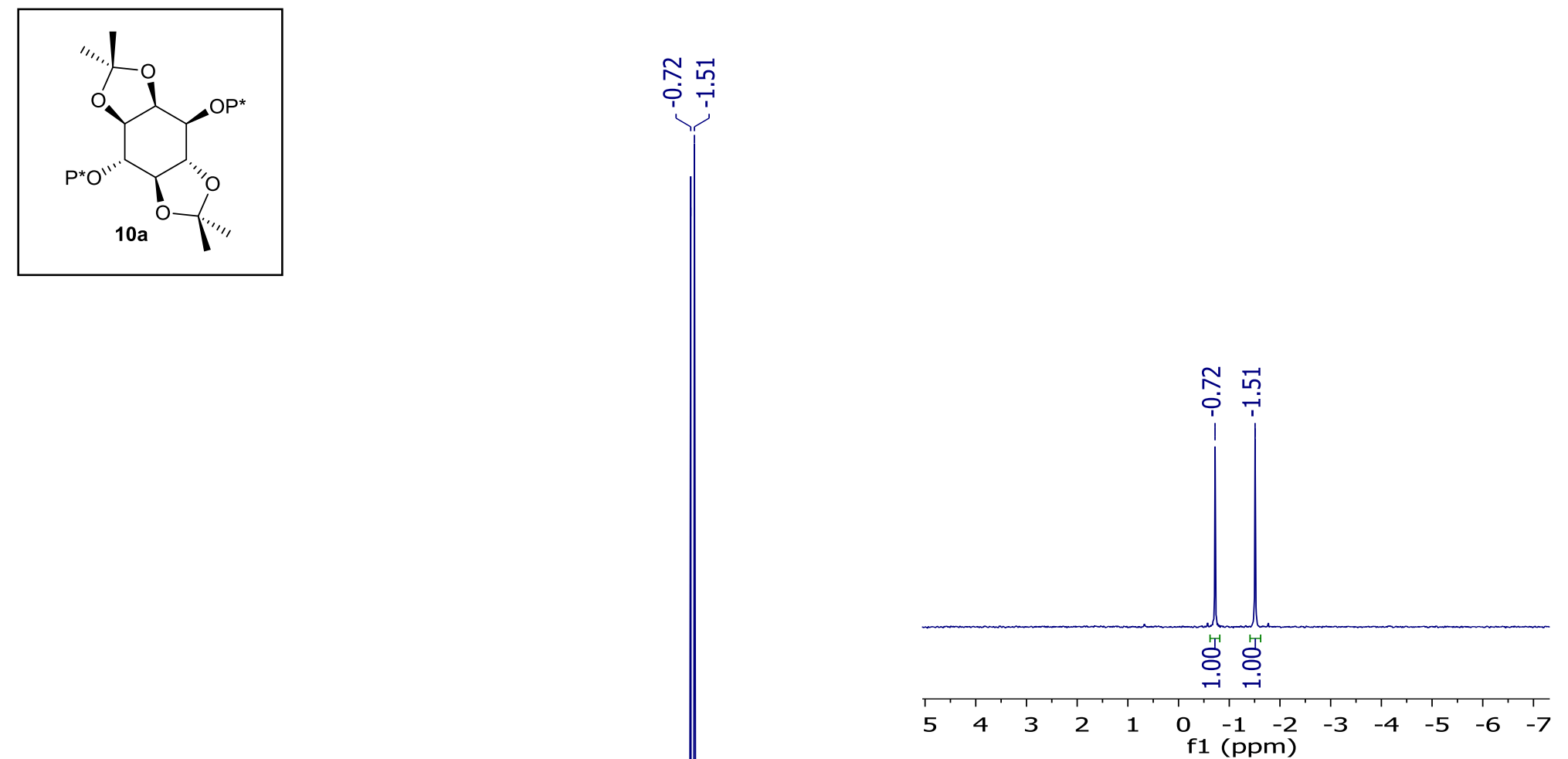

웅

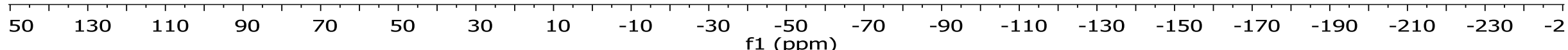


${ }^{1} \mathrm{H}$ NMR, $400 \mathrm{MHz} \mathrm{CDCl}_{3}$

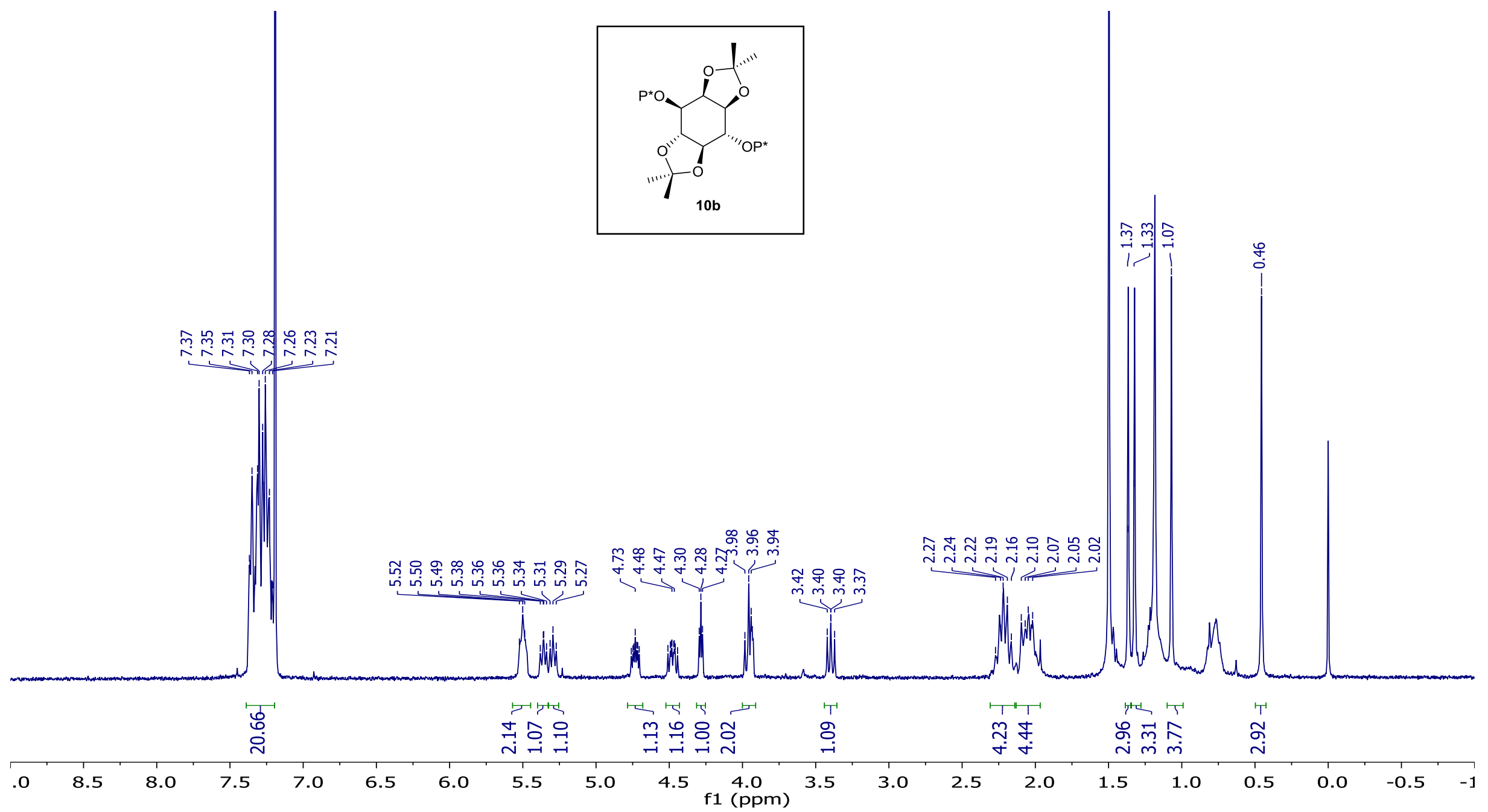




\section{${ }^{13} \mathrm{C} \mathrm{NMR}, 126 \mathrm{MHz}, \mathrm{CDCl}_{3}$}

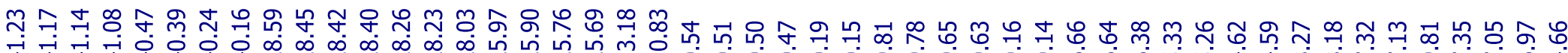

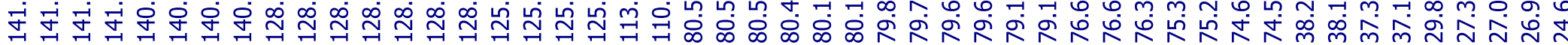
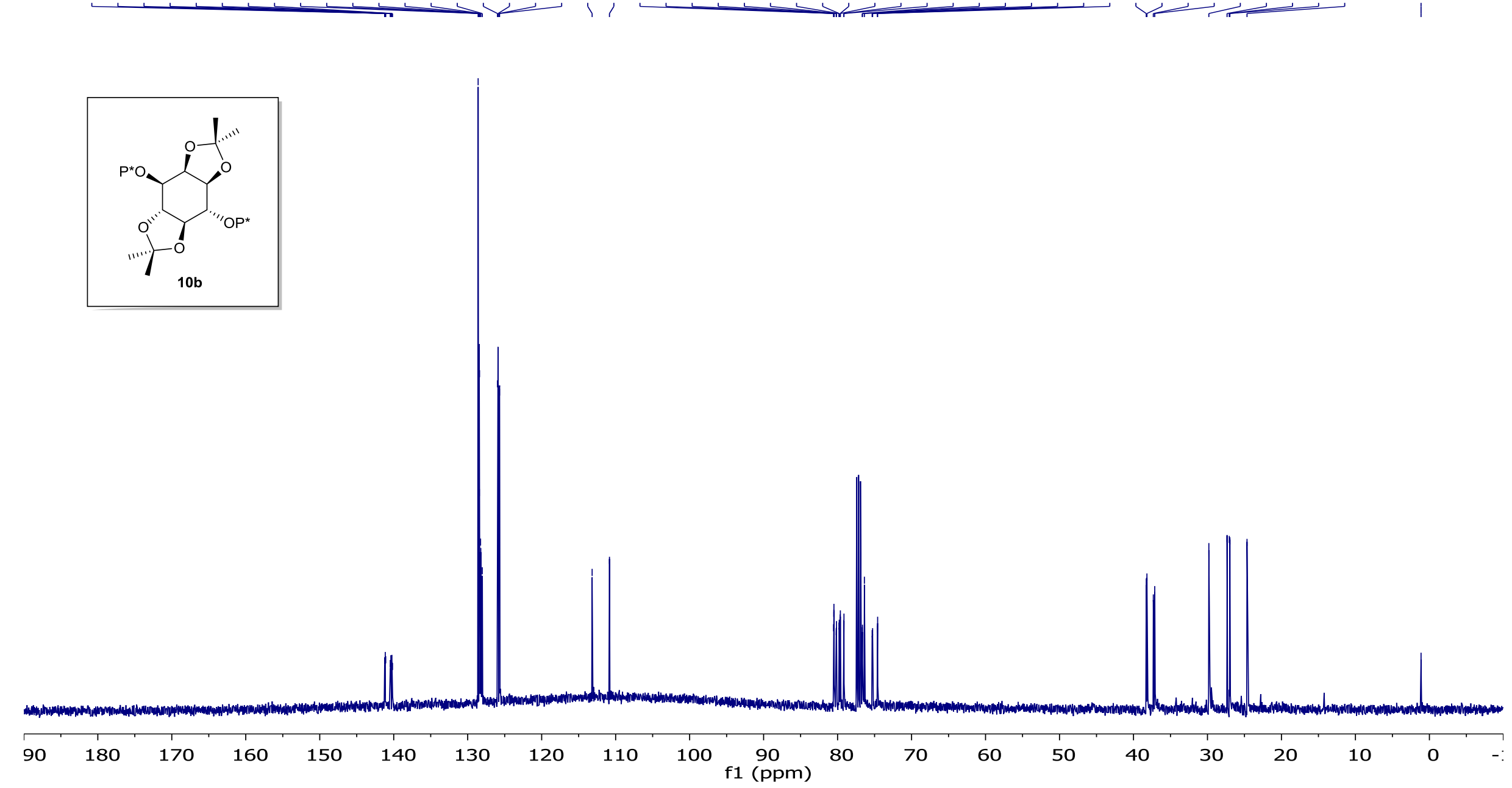
${ }^{31} \mathrm{P}$ NMR, $162 \mathrm{MHz}^{\mathrm{CDCl}}{ }_{3}$

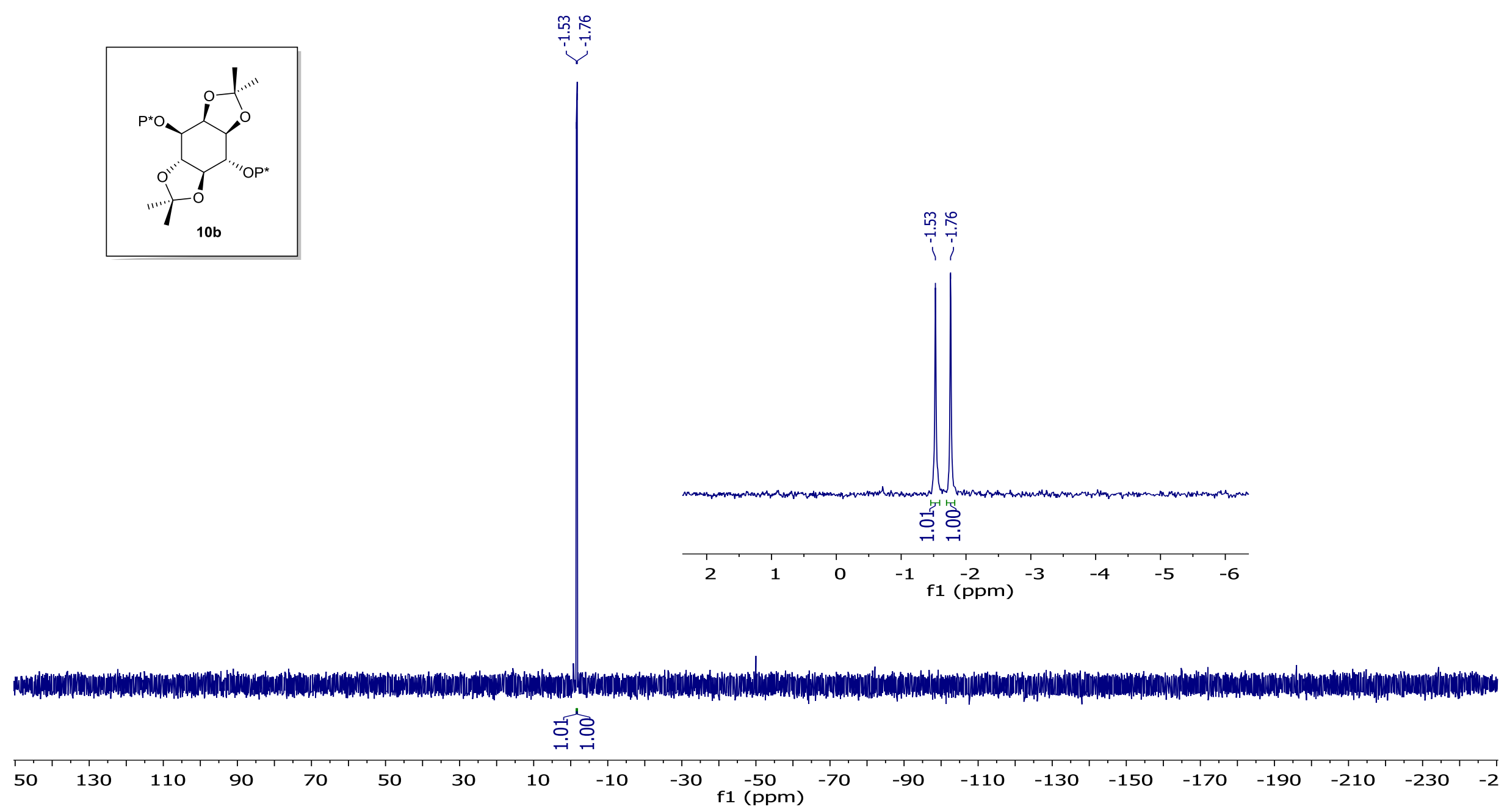




\section{${ }^{1} \mathrm{H}$ NMR, $400 \mathrm{MHz}$, MeOD}

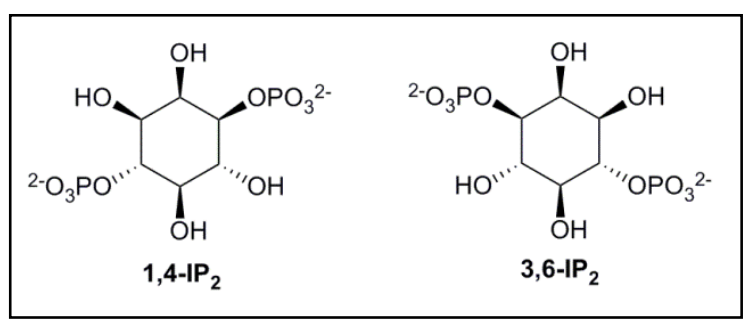

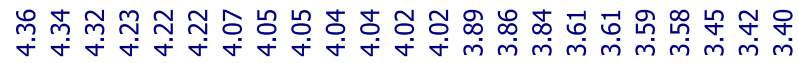

(1)

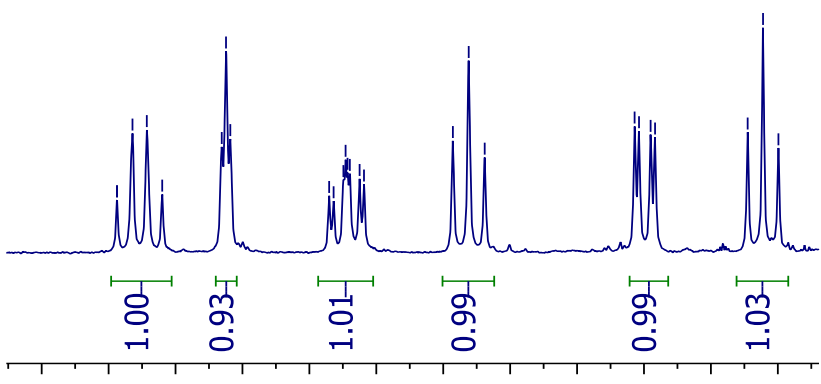

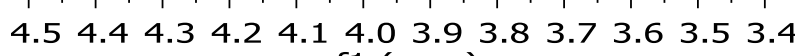
f1 (ppm)

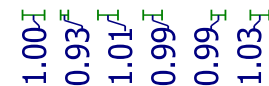

\begin{tabular}{|c|c|c|c|c|c|c|c|c|c|c|c|c|c|c|c|c|c|c|c|}
\hline .0 & 8.5 & 8.0 & 7.5 & 7.0 & 6.5 & 6.0 & 5.5 & 5.0 & 4.5 & $\begin{array}{l}4.0 \\
\mathrm{f} 1(\mathrm{ppm})\end{array}$ & 3.5 & 3.0 & 2.5 & 2.0 & 1.5 & 1.0 & 0.5 & 0.0 & -0.5 \\
\hline
\end{tabular}


${ }^{13} \mathrm{C}$ NMR, $101 \mathrm{MHz}, \mathrm{MeOD}$

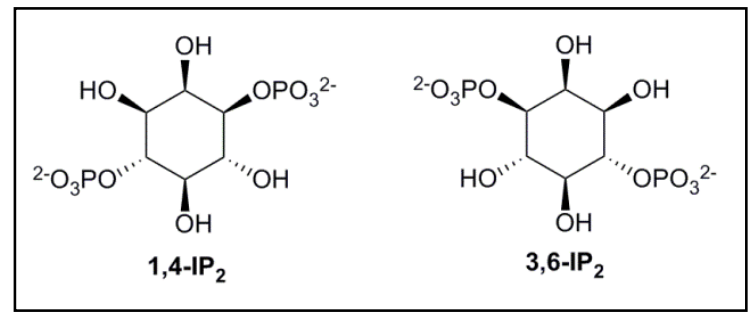

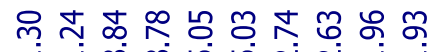

क

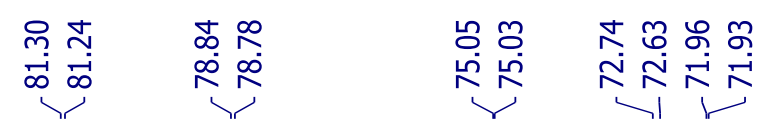

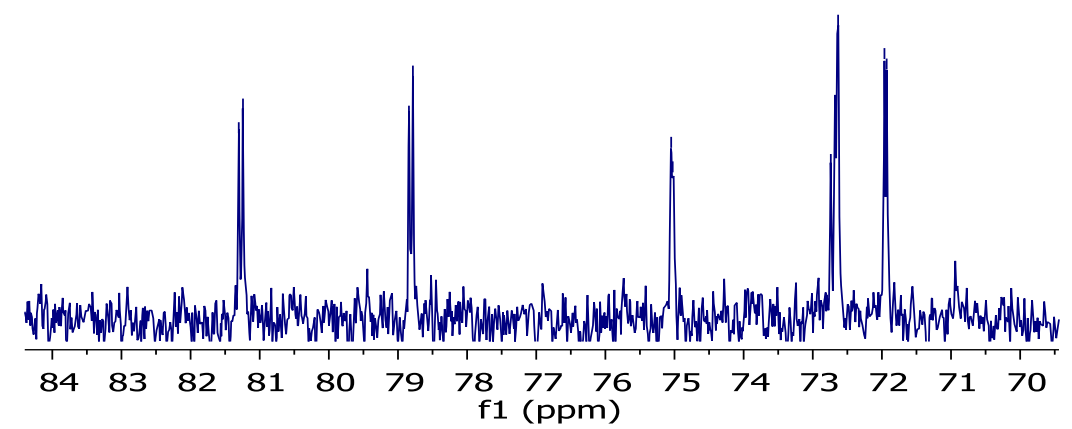

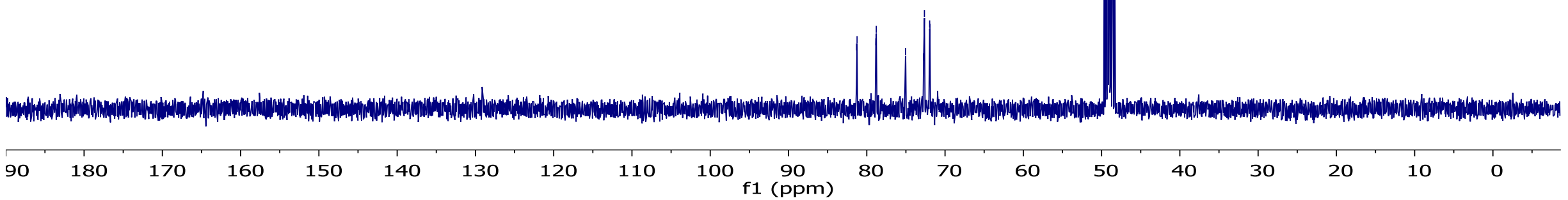


${ }^{31} \mathrm{P}$ NMR, $162 \mathrm{MHz}, \mathrm{MeOD}$

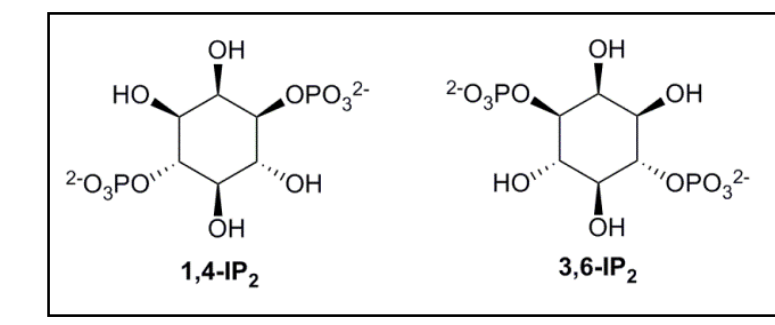

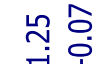

ij
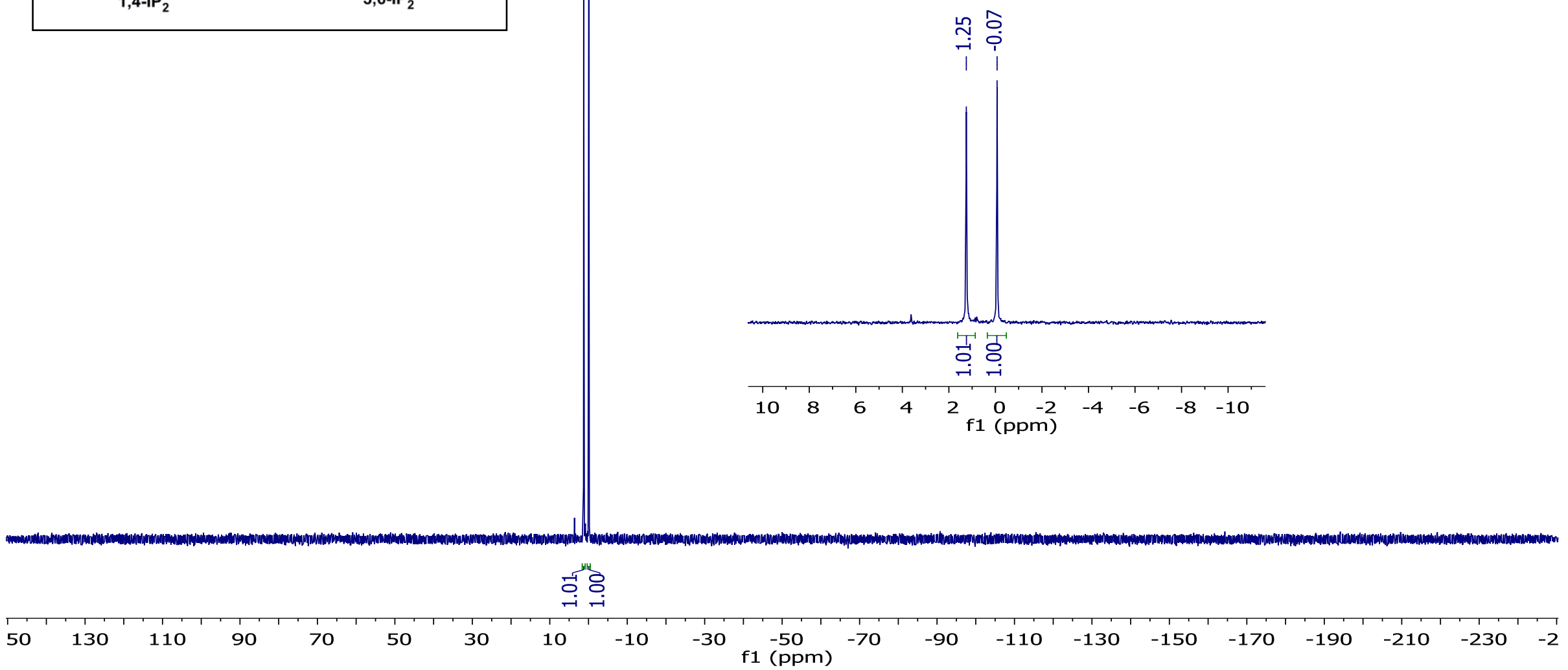


\section{${ }^{1} \mathrm{H}$ NMR, $400 \mathrm{MHz}, \mathrm{CDCl}_{3}$}

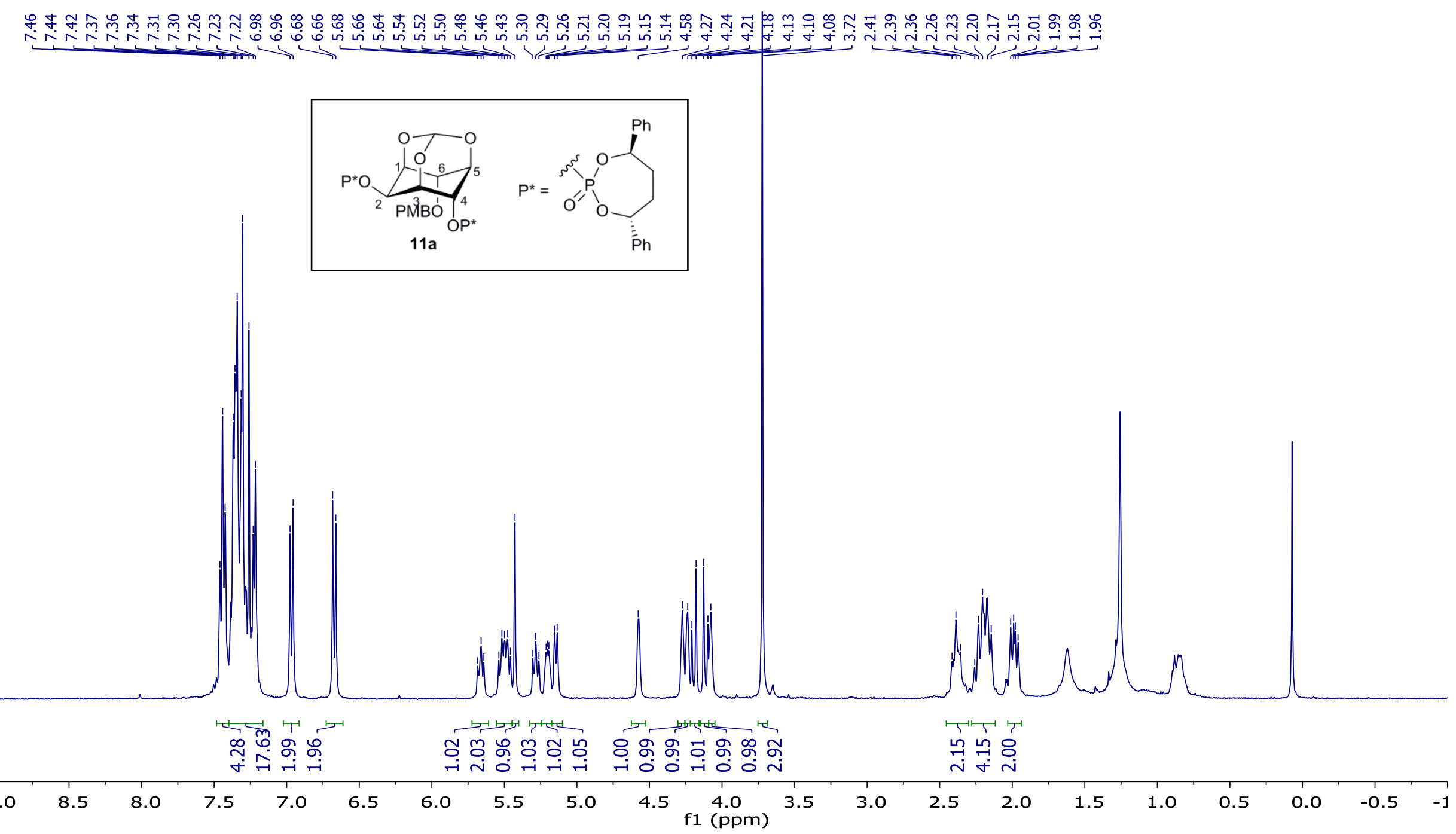


${ }^{13} \mathrm{C} N \mathrm{NR}, 101 \mathrm{MHz}, \mathrm{CDCl}_{3}$

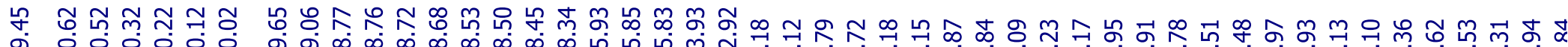

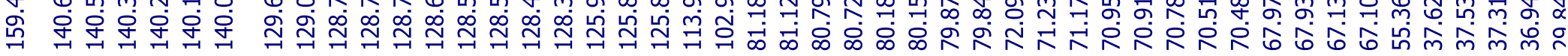
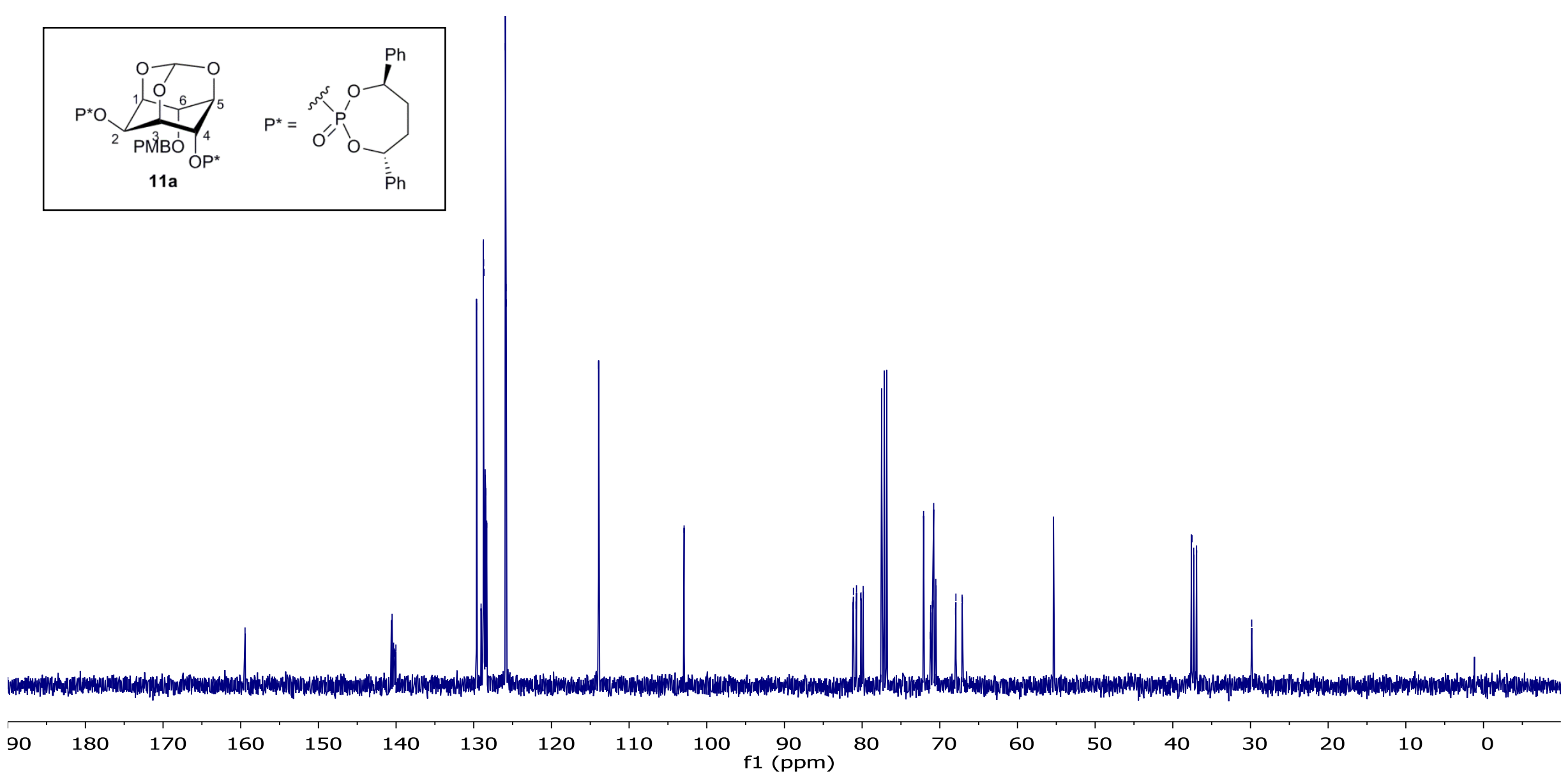
${ }^{31} \mathrm{P}$ NMR, $162 \mathrm{MHz}, \mathrm{CDCl}_{3}$

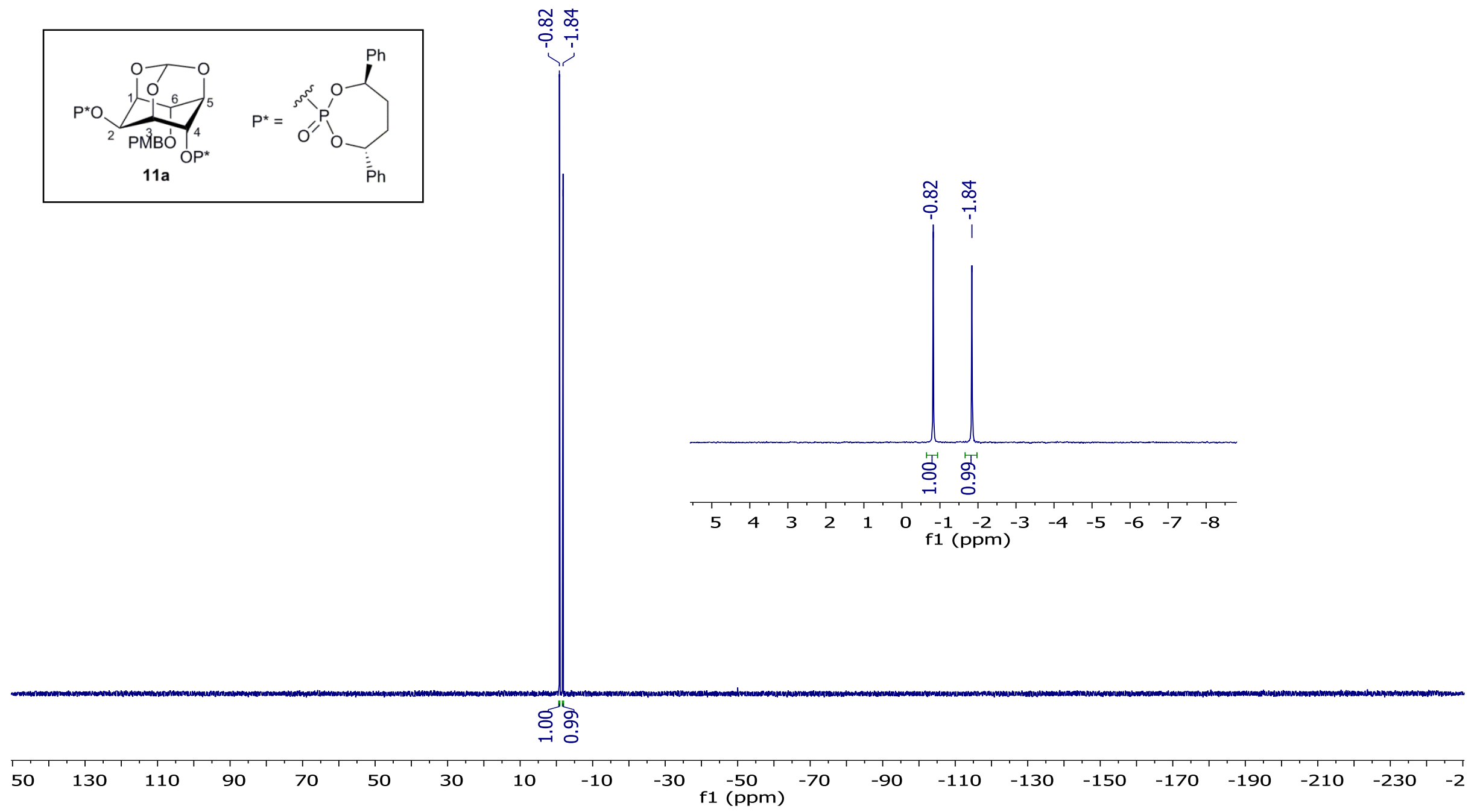




\section{${ }^{1} \mathrm{H}$ NMR, $400 \mathrm{MHz}, \mathrm{CDCl}_{3}$}

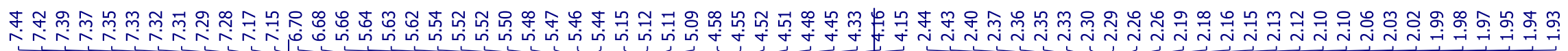

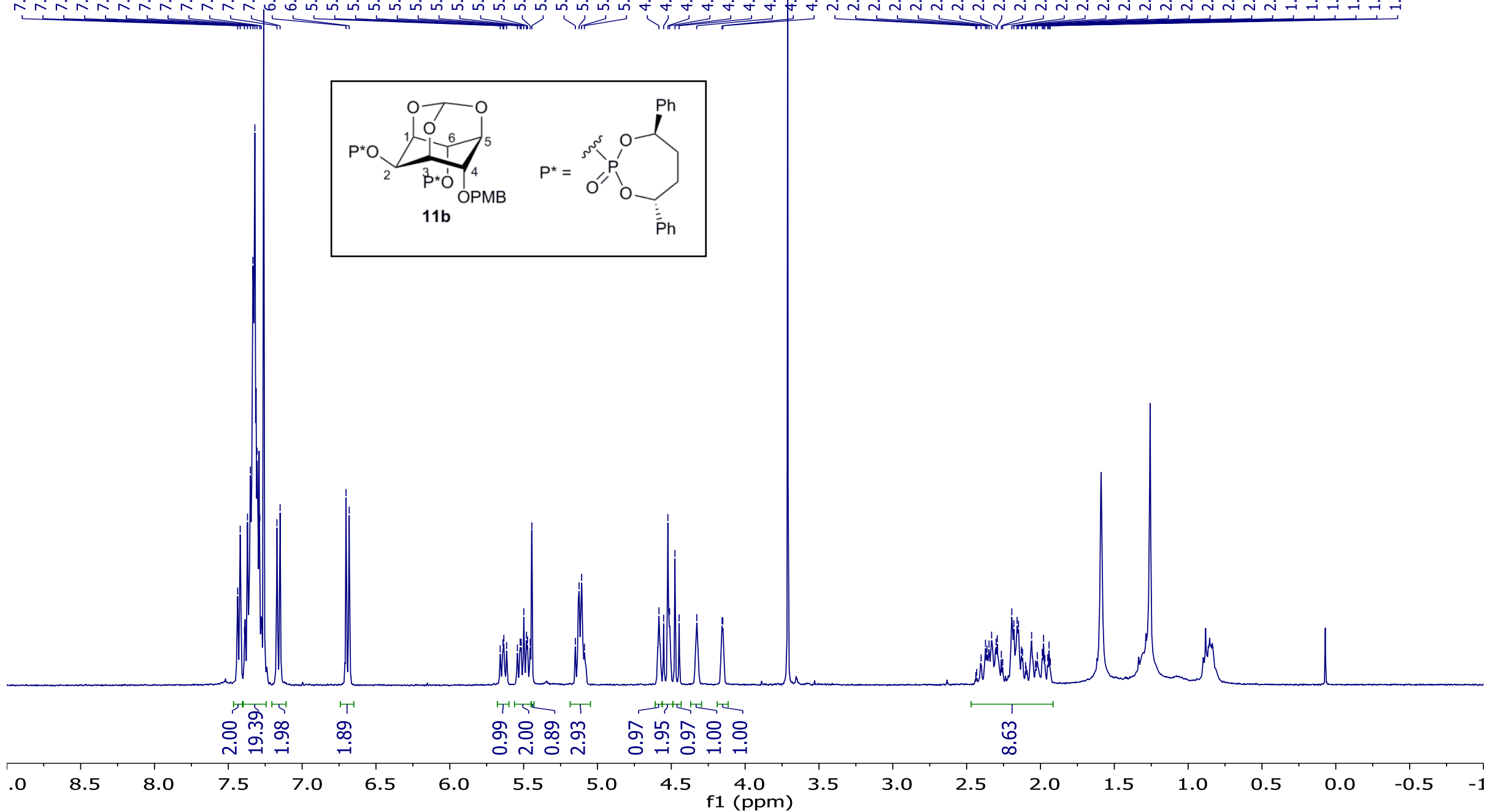


${ }^{13} \mathrm{C} \mathrm{NMR,} 126 \mathrm{MHz}, \mathrm{CDCl}_{3}$

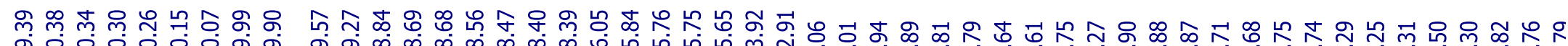

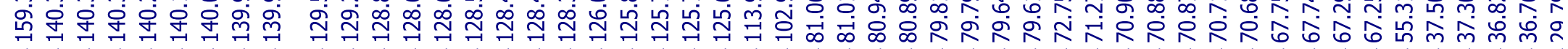

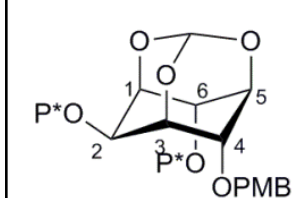

11b
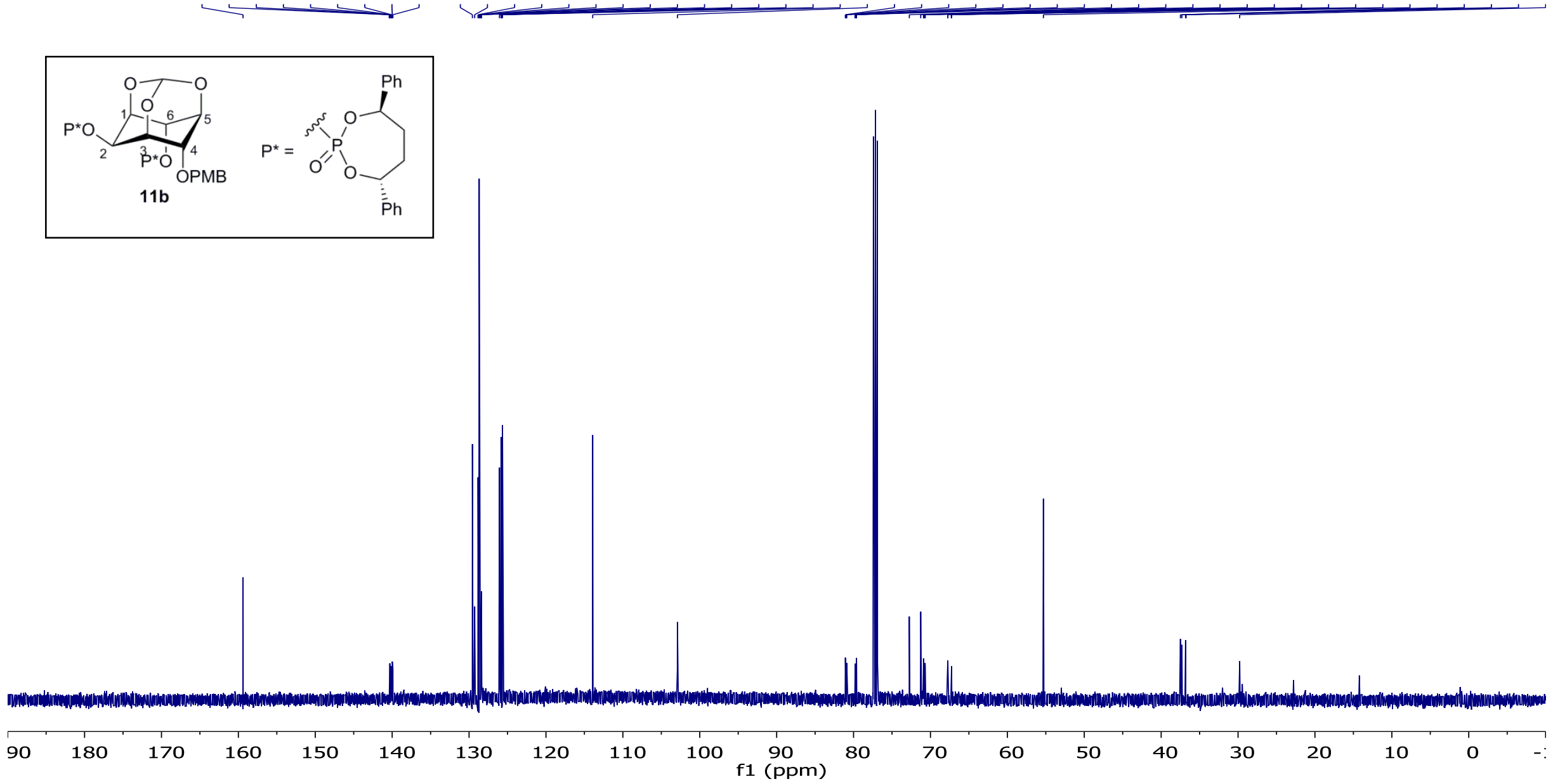


\section{${ }^{31} \mathrm{P} N M R, 162 \mathrm{MHz}, \mathrm{CDCl}_{3}$}

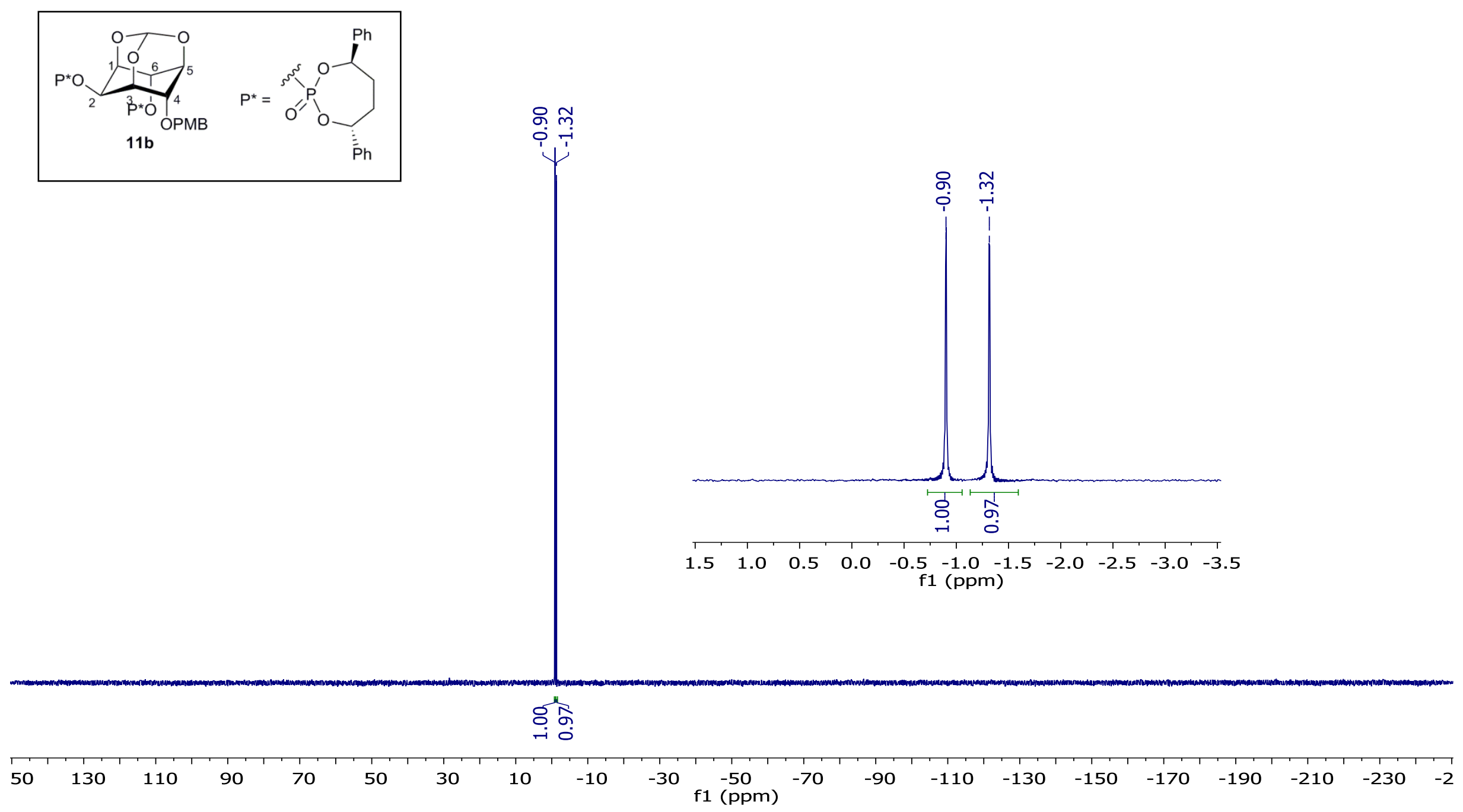




\section{${ }^{1} \mathrm{H}$ NMR, $400 \mathrm{MHz}$, MeOD}

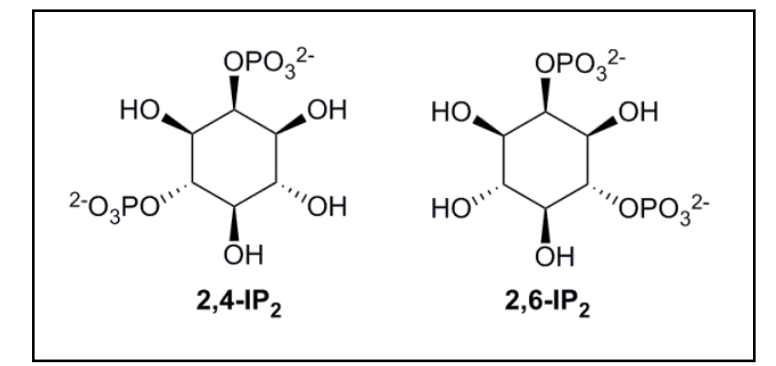

总
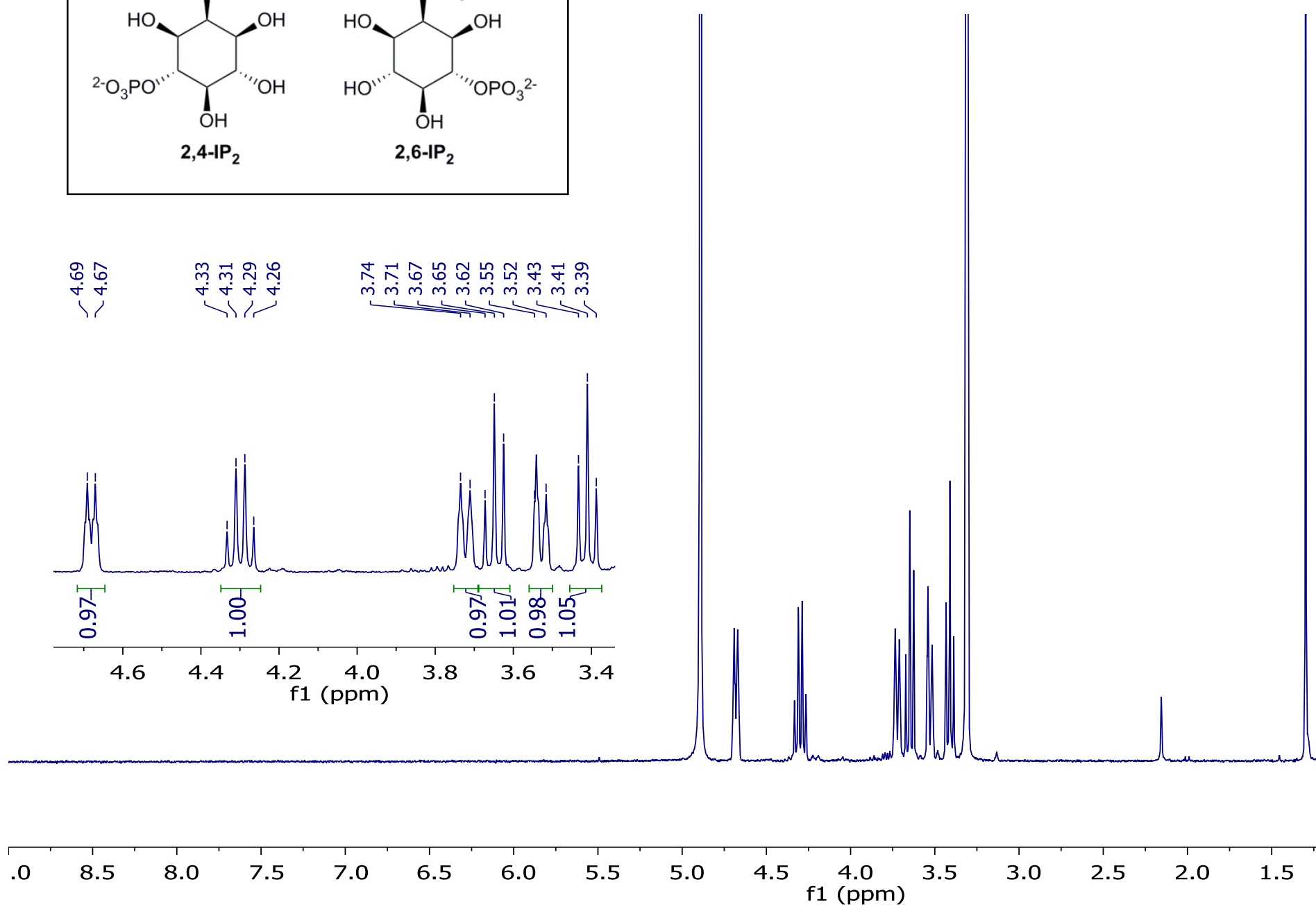


\section{${ }^{13} \mathrm{C}$ NMR, $101 \mathrm{MHz}, \mathrm{MeOD}$}

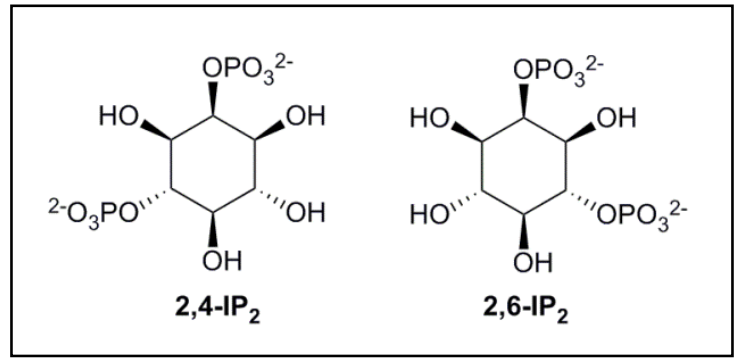

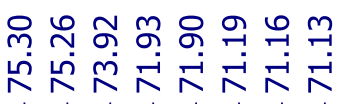

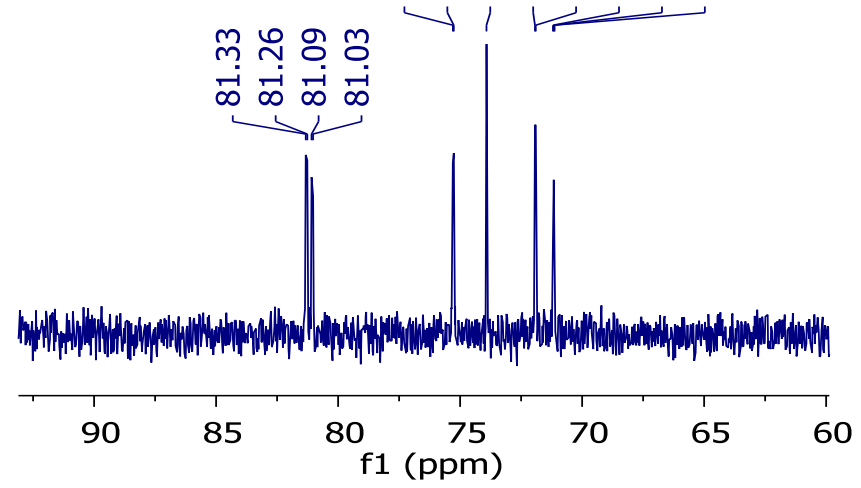

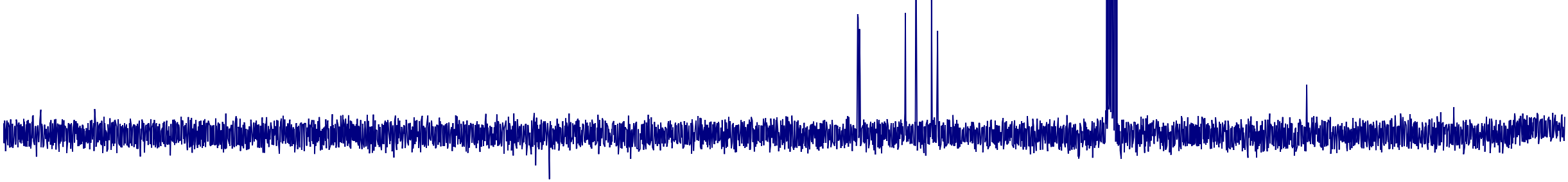

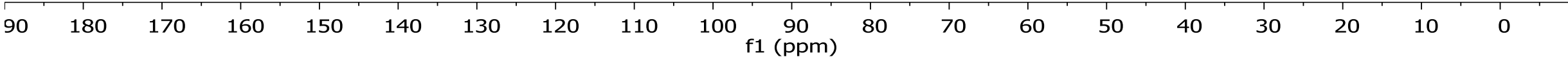


${ }^{31} \mathrm{P}$ NMR, $162 \mathrm{MHz}$, MeOD

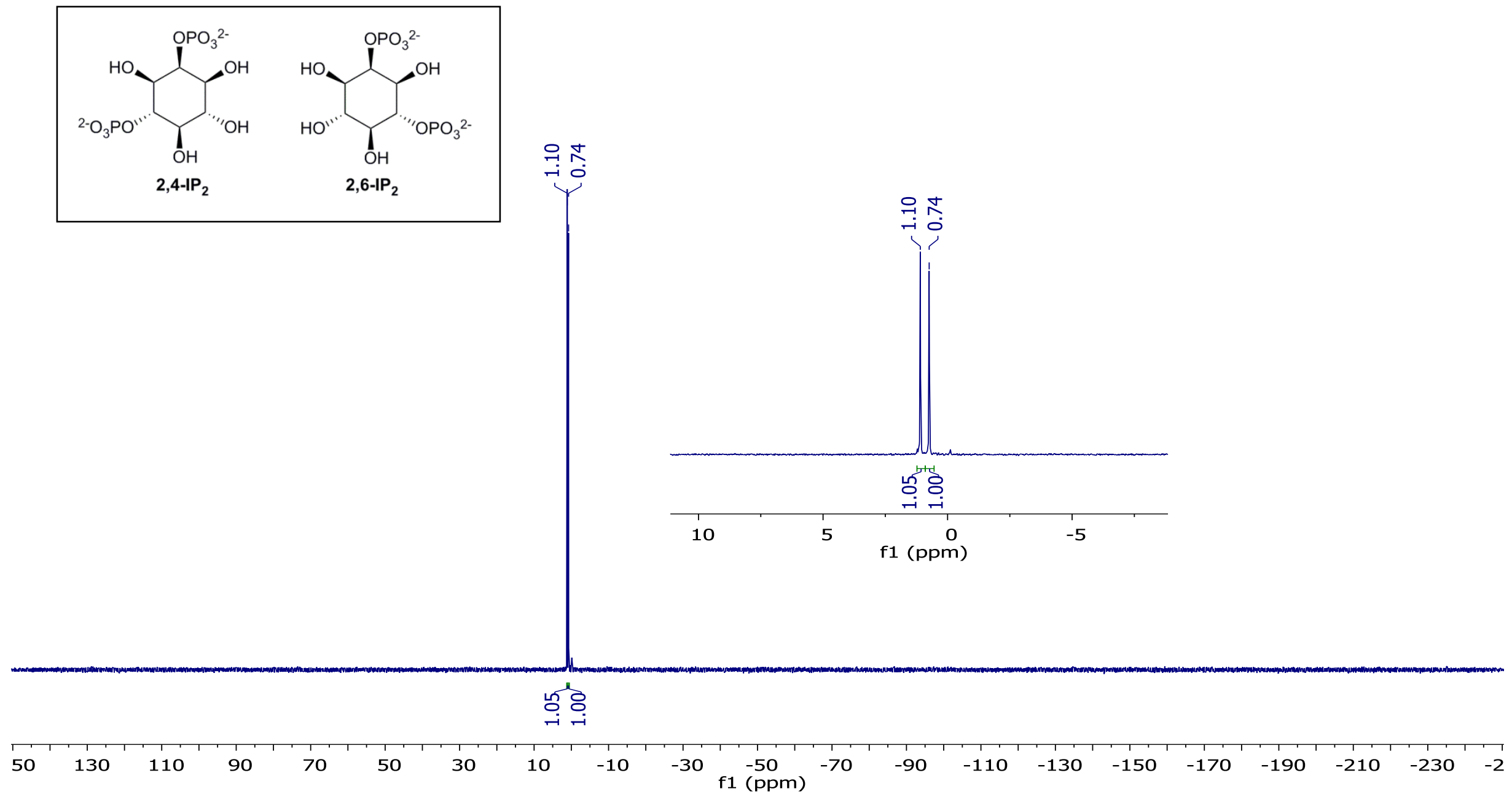




\section{${ }^{1} \mathrm{H}$ NMR, $400 \mathrm{MHz}, \mathrm{CDCl}_{3}$}

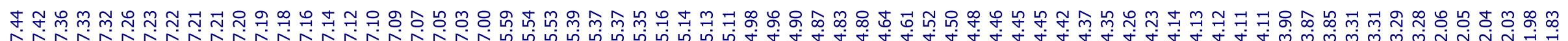

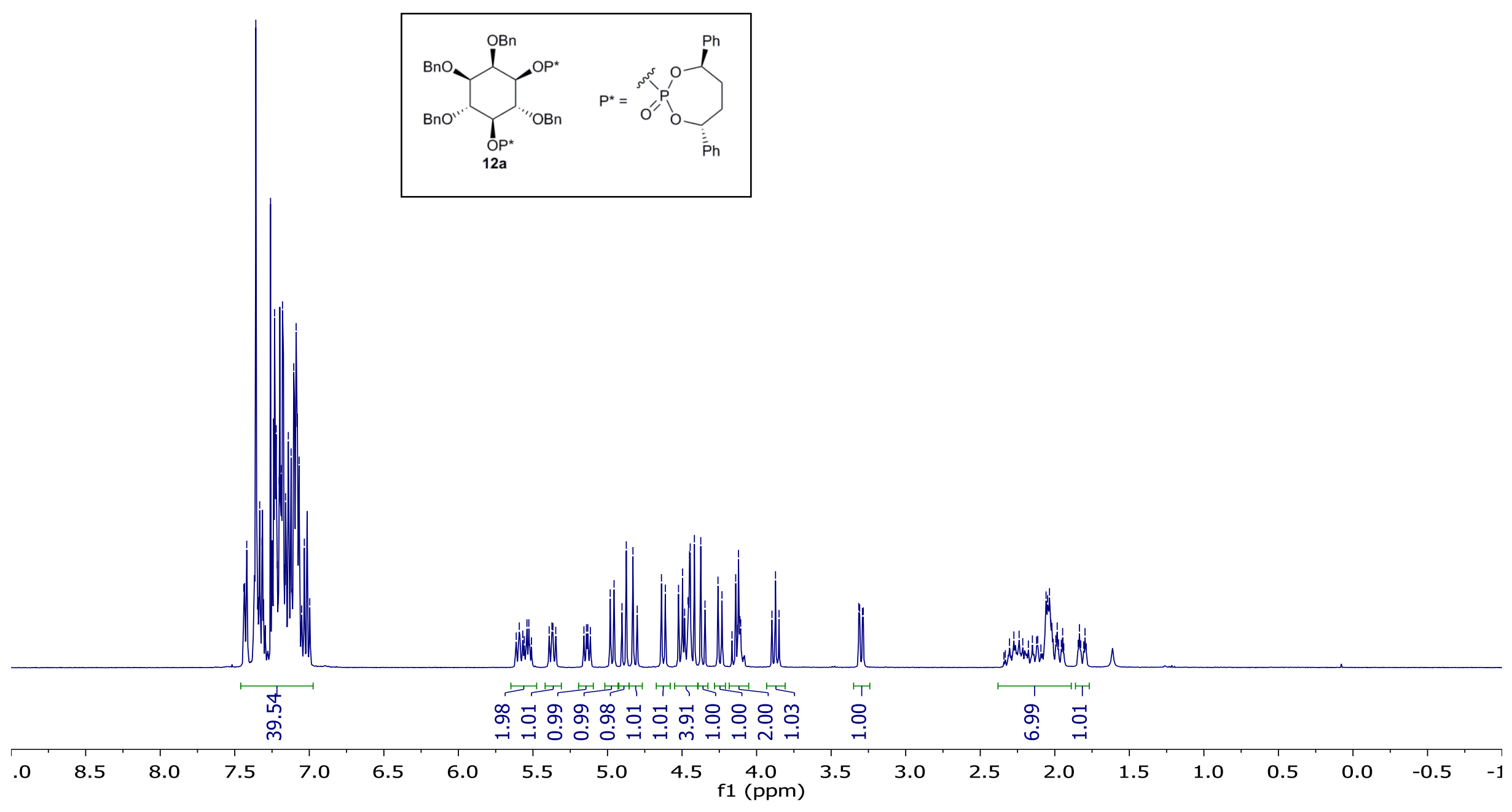




\section{${ }^{13} \mathrm{C} \mathrm{NMR}, 101 \mathrm{MHz}, \mathrm{CDCl}_{3}$}

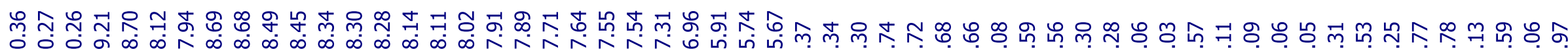

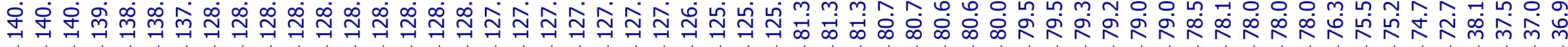

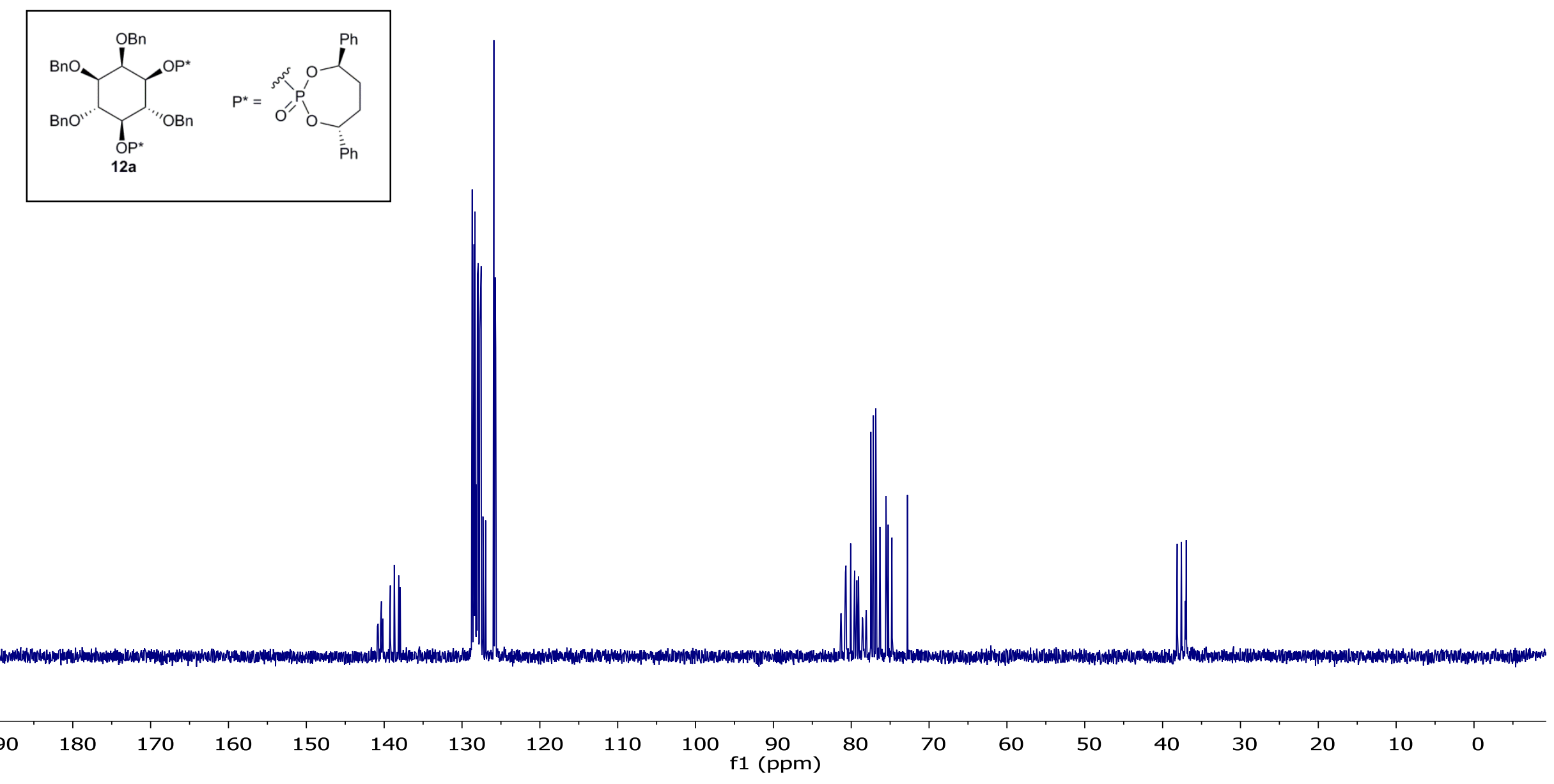


${ }^{31} \mathrm{P} N M R, 162 \mathrm{MHz}, \mathrm{CDCl}_{3}$

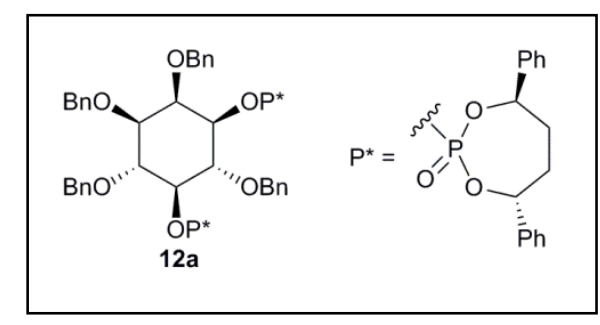

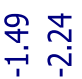

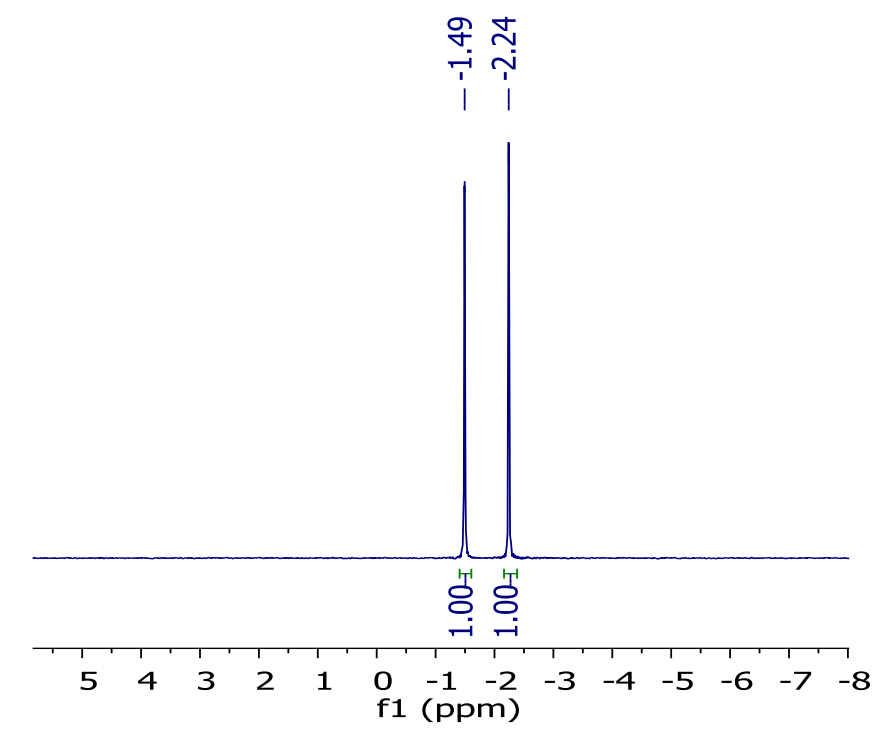

욤

$140 \quad 120 \quad 100 \quad 80 \quad 60 \quad 40,1,130$

$0+1,-20$

$-40 \quad-60$

$-80$

$-100$

$\begin{array}{llll}-120 & -140 & -160 & -180\end{array}$

$-200$

$-220$

$-240$ 


\section{${ }^{1}$ H NMR, $400 \mathrm{MHz}$, MeOD}

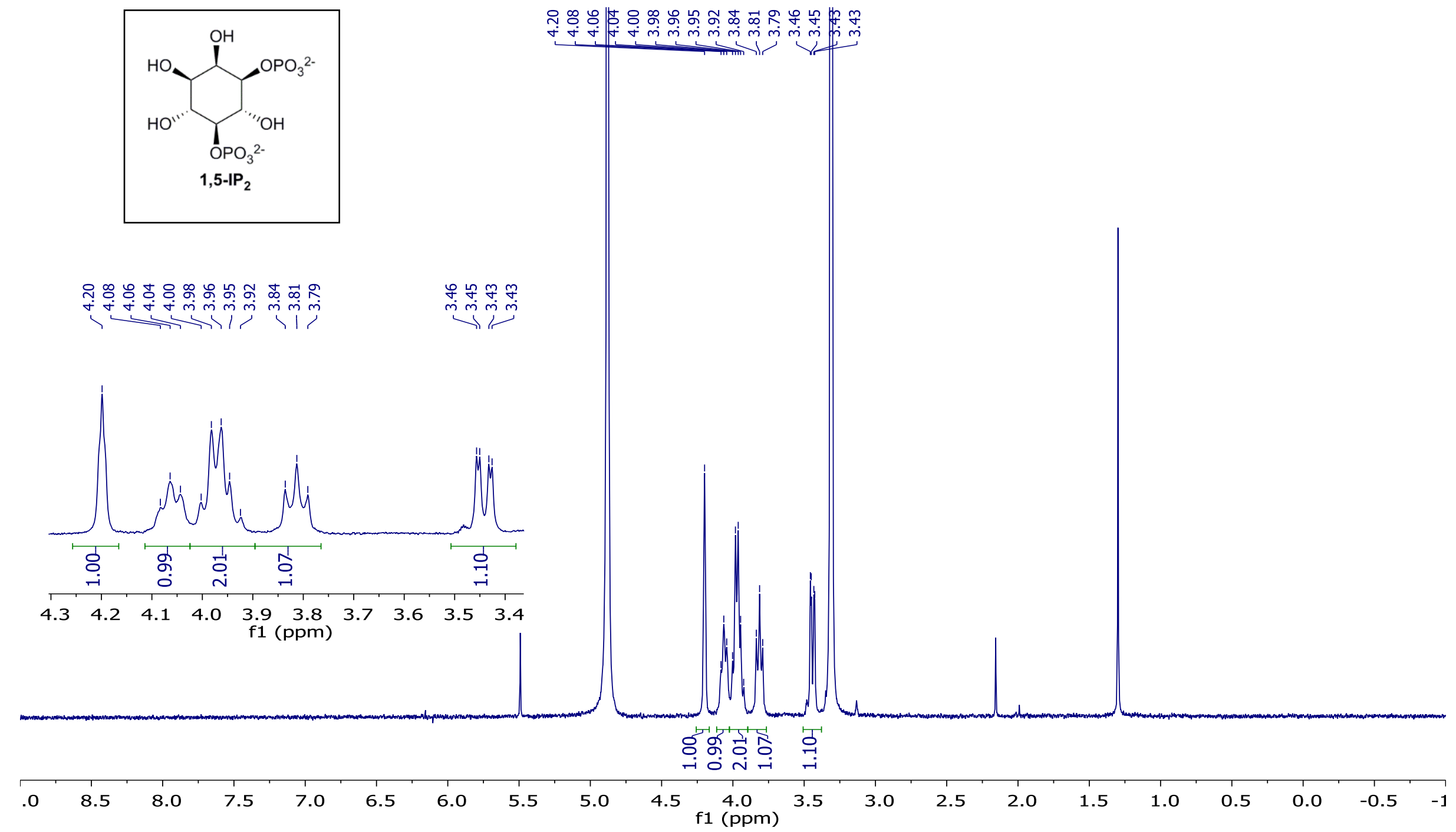

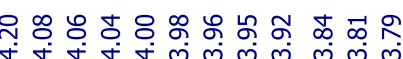

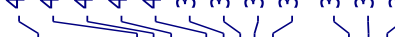
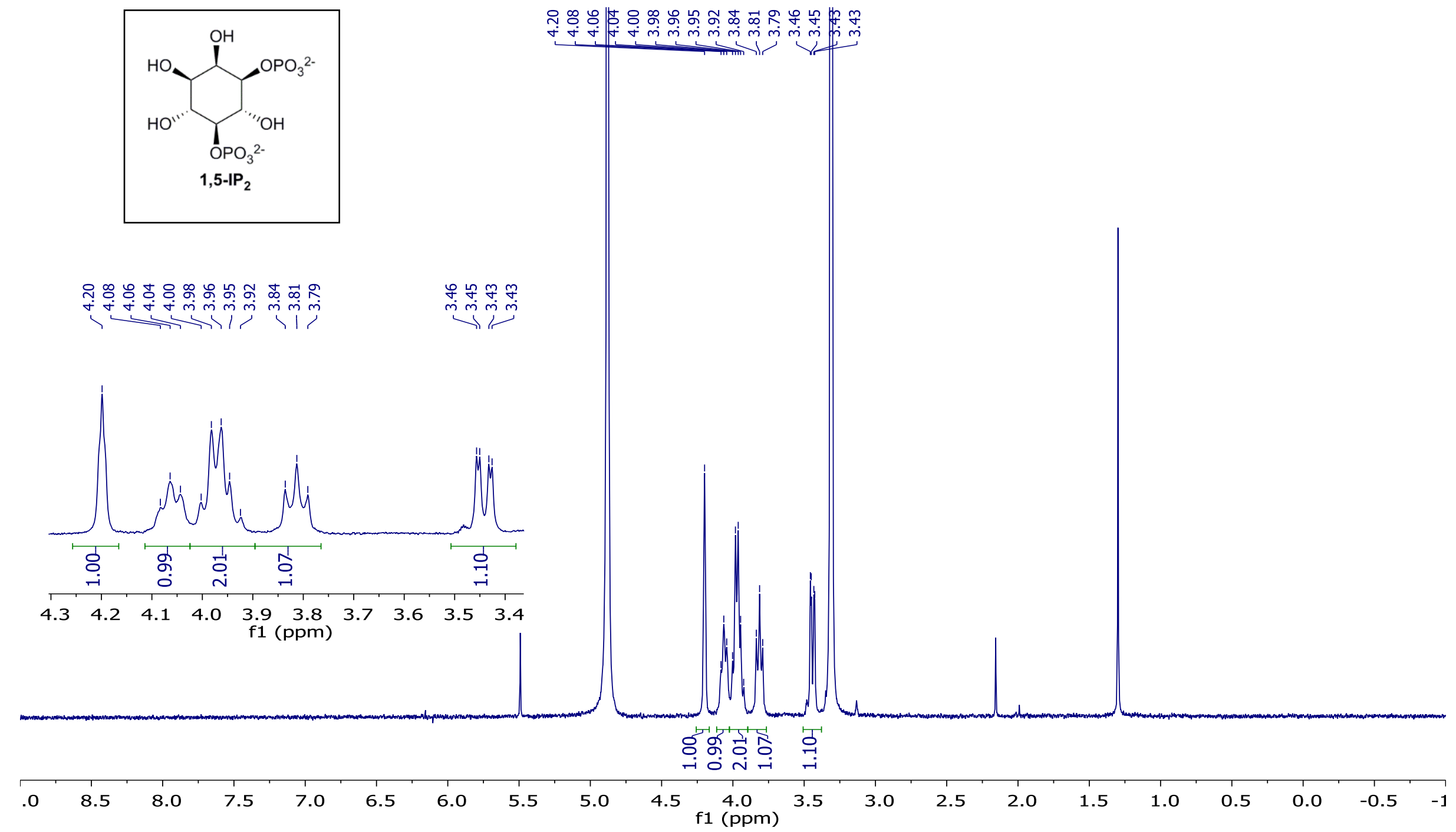

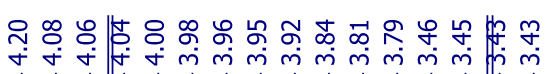




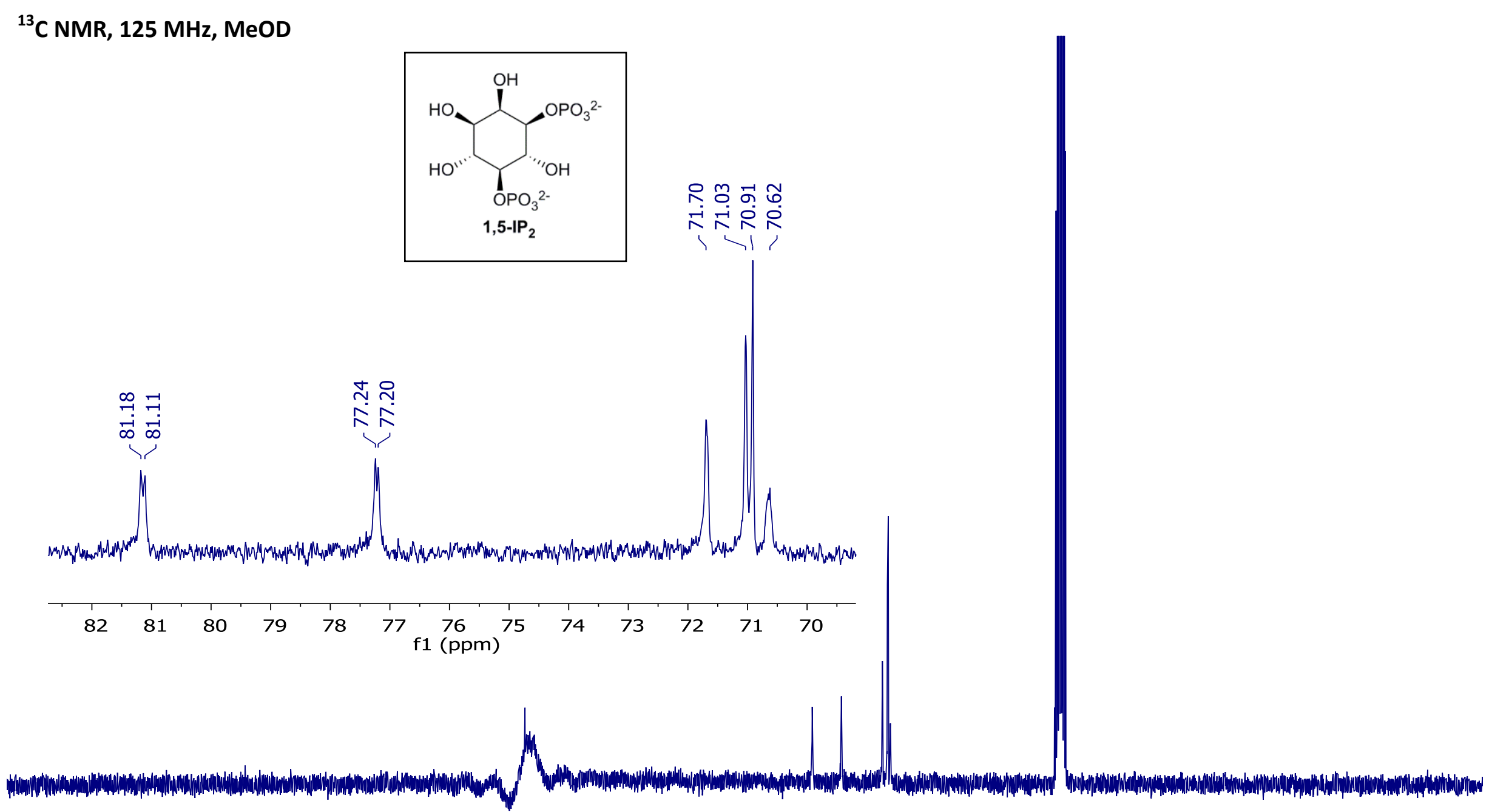




\section{${ }^{31} \mathrm{P}$ NMR, $162 \mathrm{MHz}, \mathrm{MeOD}$}

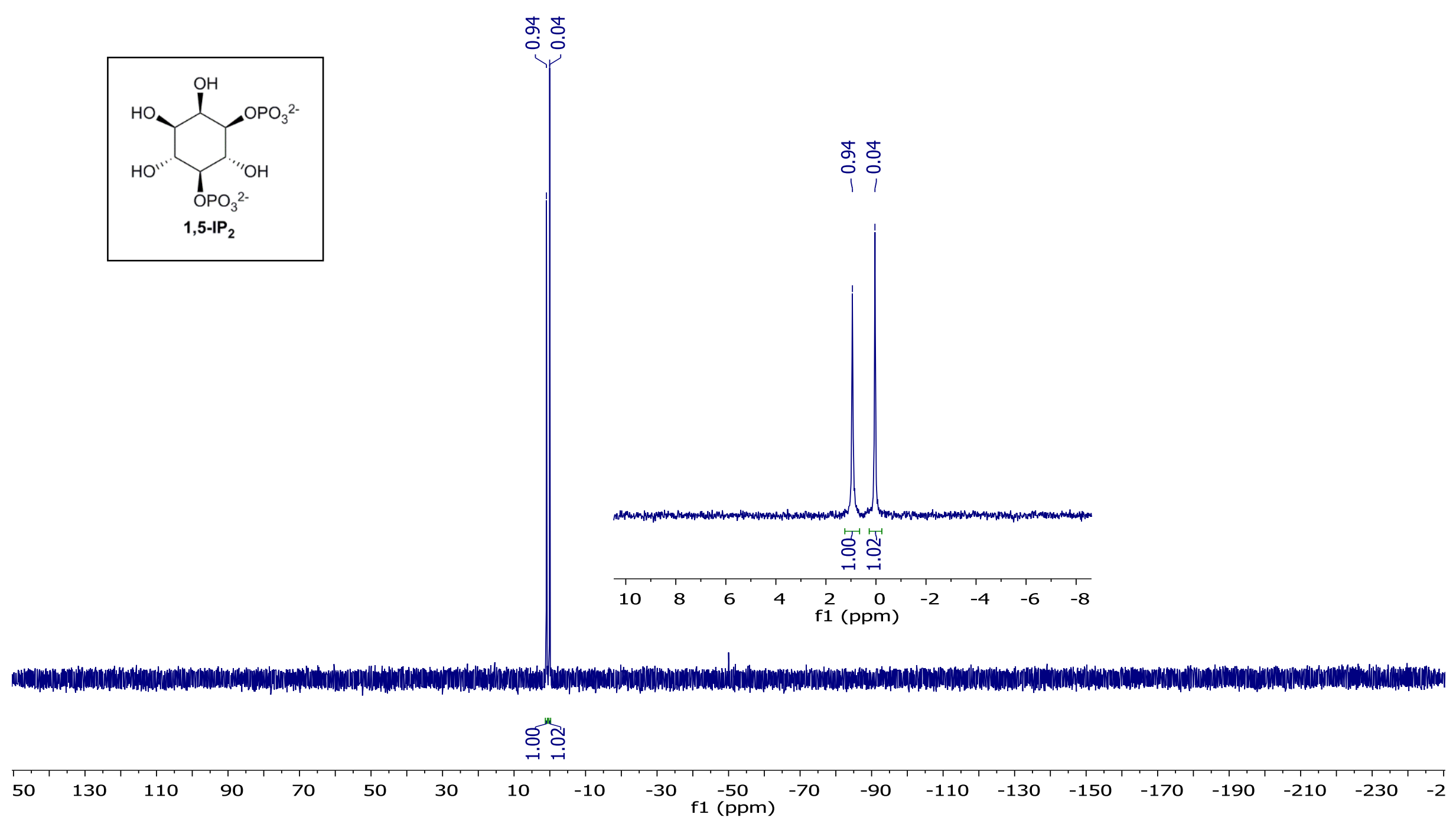

